\title{
LINEAR CODES OVER SIGNED GRAPHS
}

\author{
JOSÉ MARTÍNEZ-BERNAL, MIGUEL A. VALENCIA-BUCIO, AND RAFAEL H. VILLARREAL
}

\begin{abstract}
We give formulas, in terms of graph theoretical invariants, for the minimum distance and the generalized Hamming weights of the linear code generated by the rows of the incidence matrix of a signed graph over a finite field, and for those of its dual code. Then we determine the regularity of the ideals of circuits and cocircuits of a signed graph, and prove an algebraic formula in terms of the multiplicity for the frustration index of an unbalanced signed graph.
\end{abstract}

\section{INTRODUCTION}

The generalized Hamming weights (GHWs) of a linear code are parameters of interest in many applications [12, 16, 20, 27, 31, 37, 42, 43, 45] and they have been nicely related to the graded Betti numbers of the ideal of cocircuits of the matroid of a linear code [19, 20], to the nullity function of the dual matroid of a linear code [42], and to the enumerative combinatorics of linear codes [3, 18, 22, 23]. Because of this, their study has attracted considerable attention, but determining them is in general a difficult problem. The notion of generalized Hamming weight was introduced by Helleseth, Kløve and Mykkeltveit in [17] and was first used systematically by Wei in [42]. For convenience we recall this notion. Let $K=\mathbb{F}_{q}$ be a finite field and let $C$ be an $[m, k]$-linear code of length $m$ and dimension $k$, that is, $C$ is a linear subspace of $K^{m}$ with $k=\operatorname{dim}_{K}(C)$. Let $1 \leq r \leq k$ be an integer. Given a linear subspace $D$ of $C$, the support of $D$, denoted $\chi(D)$, is the set of nonzero positions of $D$, that is,

$$
\chi(D):=\left\{i: \exists\left(a_{1}, \ldots, a_{m}\right) \in D, a_{i} \neq 0\right\} .
$$

The $r$-th generalized Hamming weight of $C$, denoted $\delta_{r}(C)$, is given by

$$
\delta_{r}(C):=\min \left\{|\chi(D)|: D \text { is a subspace of } C, \operatorname{dim}_{K}(D)=r\right\} .
$$

As usual we call the set $\left\{\delta_{1}(C), \ldots, \delta_{k}(C)\right\}$ the weight hierarchy of the linear code $C$. The 1 st Hamming weight of $C$ is the minimum distance $\delta(C)$ of $C$, that is, one has

$$
\delta_{1}(C)=\delta(C)=\min \{\omega(\mathbf{x}): \mathbf{x} \in C \backslash\{0\}\},
$$

where $\omega(\mathbf{x})$ is the Hamming weight of the vector $\mathbf{x}$, i.e., the number of non-zero entries of $\mathbf{x}$. To determine the minimum distance is essential to find good error-correcting codes [23].

The notion of generalized Hamming weights for linear codes was extended to matroids by Britz, Johnsen, Mayhew and Shiromoto [6, p. 332] as we now explain.

Let $M$ be a matroid with ground set $E$, rank function $\rho$, nullity function $\eta$, and let $M^{*}$ be its dual matroid. The $r$-th generalized Hamming weight of $M$, denoted $d_{r}(M)$, is given by

$$
d_{r}(M):=\min \{|X|: X \subseteq E \text { and } \eta(X)=r\} \text { for } 1 \leq r \leq \eta(E) .
$$

2010 Mathematics Subject Classification. Primary 94B05; Secondary 94C15, 05C40, 05C22; $13 \mathrm{P} 25$.

Key words and phrases. Generalized Hamming weight, incidence matrix, linear code, signed graph, vector matroid, edge connectivity, frustration index, circuit, cycle, regularity, multiplicity.

The first and third authors were supported by SNI, Mexico. Second author was supported by a scholarship from CONACYT, Mexico. 
A major result of Johnsen and Verdure [19] shows that the GHWs of a matroid can be read off the minimal graded free resolution of the Stanley-Reisner ideal of the independence complex of the matroid [19, Theorem 2] (see Theorem 4.2).

We can associate to an $[m, k]$-linear code $C$ the vector matroid $M[A]$ on the ground set $E=\{1, \ldots, m\}$, where $A$ is a generator matrix of $C$. The rank function (resp. nullity) of $M[A]$ is given by $\rho(X)=\operatorname{rank}\left(A_{X}\right)$ (resp. $\left.\eta(X)=|X|-\rho(X)\right)$ for $X \subseteq E$, where $A_{X}$ is the submatrix of $A$ obtained by picking the columns indexed by $X$. It can be verified that the matroid $M[A]$ does not depend on the generator matrix we choose. We call $M[A]$ the matroid of $C$. If $H$ is a parity check matrix of $C$, then $M[A]^{*}=M[H]$ and $M[A]=M[H]^{*}$. By Lemma 2.4, one has

$$
\delta_{r}\left(C^{\perp}\right)=d_{r}(M[A]) \text { for } 1 \leq r \leq m-k \text { and } \delta_{r}(C)=d_{r}\left(M[A]^{*}\right) \text { for } 1 \leq r \leq k .
$$

Thus computing GHWs of vector matroids is equivalent to computing those of linear codes. This relationship between the GHWs of linear codes and those of vector matroids is attributed to Wei [42, Theorem 2] (cf. Theorem 2.2). In this work we study GHWs of linear codes defined over signed graphs, combining the theory of GHWs of matroids [4, 6, 19, 20] and the combinatorial structure of signed-graphic matroids [46, 47, 48, that we introduce next.

A signed graph $G_{\sigma}$ is a pair $(G, \sigma)$ consisting of a multigraph $G$ with vertex set $V(G)$ and edge set $E(G)$ (loops and multiple edges are permitted), and a mapping $\sigma: E(G) \rightarrow\{ \pm\}$, that assigns a sign to each edge. If no loops or multiple edges are permitted, $G$ is called a simple graph and $G_{\sigma}$ is called a signed simple graph. In particular, the signed graph with $\sigma(e)=+$ (resp. $\sigma(e)=-$ ) for all $e$, denoted $G_{+}$(resp. $G_{-}$), is called the positive signed graph (resp. negative signed graph) on $G$. There are more general definitions of signed graphs, where the edge set includes empty loops and half edges, that are essential to represent root systems [46].

Let $G_{\sigma}$ be a signed graph. A cycle of $G_{\sigma}$ is a simple closed path in $G$. A cycle with an even number of negative edges is called balanced. A signed graph is balanced if every cycle is balanced. An isolated vertex is regarded as balanced. A bowtie of $G_{\sigma}$ is the union of two unbalanced cycles which meet at a single vertex or the union of two vertex-disjoint unbalanced cycles and a simple path which meets one cycle at each end and is otherwise disjoint from them.

A central result of Zaslavsky [46, Theorem 5.1] shows the existence of a matroid $M\left(G_{\sigma}\right)$ with ground set $E(G)$, called the signed-graphic matroid of $G_{\sigma}$, whose rank function is

$$
\rho(X)=|V(G)|-c_{0}(X) \text { for } X \subseteq E(G),
$$

where $c_{0}(X)$ is the number of balanced connected components of the signed subgraph with edge set $X$ and vertex set $V(G)$. The circuits of $M\left(G_{\sigma}\right)$ are the balanced cycles and the bowties of $G_{\sigma}$. The circuits of $M\left(G_{\sigma}\right)$ are called the circuits of $G_{\sigma}$.

If $G_{\sigma}=G_{+}$, the signed-graphic matroid $M\left(G_{+}\right)$is the graphic matroid $M(G)$ of $G$ whose circuits are the cycles of $G\left[28\right.$, 44. If $G_{\sigma}=G_{-}$, the signed-graphic matroid $M\left(G_{-}\right)$is the even cycle matroid [46] whose circuits are the even cycles and the bowties of $G_{-}$. The circuits of the matroids $M\left(G_{+}\right), M\left(G_{-}\right)$and those of their dual matroids - as well as the related notion of an elementary integral vector-occur in coding theory [9, 34, convex analysis [29], the theory of toric ideals of graphs [2, 11, 25, 32, 39, 40, and in matroid theory [28, 33, 46, 49].

The content of this paper is as follows. In Section 2 we briefly introduce matroids and present some well known results about GHWs of matroids and linear codes.

In what follows $G_{\sigma}$ denotes a signed graph with $s$ vertices, $m$ edges, $c$ connected components, and $c_{0}$ balanced components, and $K$ denotes a finite field $\mathbb{F}_{q}$ of characteristic $p$. The incidence matrix code of $G_{\sigma}$ over the field $K$, denoted by $C$, is the linear code generated by the row vectors of the incidence matrix of $G_{\sigma}$ (Definition 3.6). In Section 3 we present our main results on the 
generalized Hamming weights of incidence matrix codes of signed graphs and those of their dual codes, and describe the GHWs of the signed-graphic matroid of a signed graph and those of its dual matroid, in terms of the combinatorics of the signed graph.

The frustration index of $G_{\sigma}$, denoted $\varphi\left(G_{\sigma}\right)$, is the smallest number of edges whose deletion from $G_{\sigma}$ leaves a balanced signed graph. The minimum distance of $C$ is bounded from above by $\varphi\left(G_{\sigma}\right)$ if $p \neq 2$ (Remark 5.5). We are interested in the following related invariant. The $r$-th cogirth of $G_{\sigma}$, denoted $v_{r}\left(G_{\sigma}\right)$, is the minimum number of edges whose removal results in a signed graph with $r$ balanced components. If $r=1$ and $G_{\sigma}$ is connected, $v_{1}\left(G_{\sigma}\right)$ is the cogirth of $M\left(G_{\sigma}\right)$, that is, the minimum size of a cocircuit of $M\left(G_{\sigma}\right)$ (Lemma 3.4). We denote $v_{1}\left(G_{\sigma}\right)$ simply by $v\left(G_{\sigma}\right)$. The $r$-th edge connectivity of $G_{\sigma}$, denoted $\lambda_{r}\left(G_{\sigma}\right)$ or $\lambda_{r}(G)$, is the minimum number of edges whose removal results in a signed graph with $r+1$ connected components. Note that the $r$-th edge connectivity is a property of the underlying multigraph $G$, that is, it is independent of $\sigma$. If $r=1, \lambda_{1}\left(G_{\sigma}\right)$ is the edge connectivity of $G_{\sigma}$ and is denoted by $\lambda\left(G_{\sigma}\right)$. We will relate these graph invariants to the generalized Hamming weights and the minimum distance of incidence matrix codes.

Our main results on linear codes are the following. First, we give graph theoretical formulas for the generalized Hamming weights of the incidence matrix code of a signed graph.

Theorem 3.16 If $C$ is the incidence matrix code of a connected signed graph $G_{\sigma}$, then

$$
\delta_{r}(C)= \begin{cases}v_{r}\left(G_{\sigma}\right) & \text { if } p \neq 2, G_{\sigma} \text { is unbalanced and } 1 \leq r \leq s, \\ \lambda_{r}(G) & \text { if } p=2 \text { and } 1 \leq r \leq s-1, \\ \lambda_{r}(G) & \text { if } G_{\sigma} \text { is balanced and } 1 \leq r \leq s-1 .\end{cases}
$$

We show that the formulas of [26, Corollary 2.13] for the generalized Hamming weights of incidence matrix codes of simple graphs can be extended to multigraphs (Corollary 3.17). Then we show combinatorial formulas for the minimum distance of the incidence matrix code of a signed graph [28, Proposition 9.2.4] (Corollary 3.18).

A family of circuits $\left\{C_{i}\right\}_{i=1}^{r}$ of a matroid $M$ is called non-redundant if $\bigcup_{i \neq j} C_{i} \subsetneq \bigcup_{i=1}^{r} C_{i}$ for $j=1, \ldots, r$ [4, 7]. Our next result gives graph theoretical formulas for the generalized Hamming weights of the dual code of the incidence matrix code of a signed graph. Part (a) extends the analogous result for graphs of Britz [4, Section 3].

Theorem 3.19 Let $C$ be the incidence matrix code of a connected signed graph $G_{\sigma}$.

(a) If $p=2$ or $G_{\sigma}$ is balanced, and $1 \leq r \leq m-s+1$ (resp. $\left.1 \leq r \leq s-1\right)$, then $\delta_{r}\left(C^{\perp}\right)$ (resp. $\delta_{r}(C)$ ) is the minimum number of edges of $G$ forming a union of $r$ non-redundant cycles (resp. cocycles) of $G$.

(b) If $p \neq 2$ and $1 \leq r \leq m-s$ (resp. $1 \leq r \leq s)$, then $\delta_{r}\left(C^{\perp}\right)\left(\right.$ resp. $\left.\delta_{r}(C)\right)$ is the minimum number of edges of $G$ forming a union of $r$ non-redundant balanced cycles and bowties (resp. cocircuits) of $G_{\sigma}$.

If $C$ is the incidence matrix code of a connected digraph $\mathcal{D}$ and $G$ is its underlying multigraph, we show that $\delta_{r}(C)=\lambda_{r}(G)$ and give graph theoretical formulas for the generalized Hamming weights of the dual code $C^{\perp}$ (Corollary 3.20). For a connected multigraph, we give formulas for the GHWs of the dual of its incidence matrix code (Corollary 3.21).

The main result of Section 4 gives explicit formulas for the regularity of the ideals of circuits and cocircuits of the vector matroid of the incidence matrix of a signed graph (Theorem 4.7). This invariant is a measure for the complexity of the minimal graded free resolution of these ideals and has been used to study polynomial interpolation problems [10]. 
Let $M$ be the matroid of $C$. By Theorems 3.16 and 3.19, one has graph theoretical formulas for the weight hierarchies of $C$ and $C^{\perp}$. On the other hand, using Macaulay2 [14, the package Matroids [8], and the formulas of Johnsen and Verdure (Theorem 4.2, Corollary 4.3), we can compute the weight hierarchies of $C$ and $C^{\perp}$. Hence, our results can be used to compute the $r$-th cogirth $v_{r}\left(G_{\sigma}\right)$ of $G_{\sigma}$ and the $r$-th edge connectivity $\lambda_{r}\left(G_{\sigma}\right)$ of $G_{\sigma}$. The main result of Section $\left[5\right.$ is an algebraic formulation for the frustration index of $G_{\sigma}$-in terms of the degree or multiplicity of graded ideals - that can be used to compute or estimate this number using Macaulay2 [14] (Theorem 5.4, Example 6.6). If $G$ is a graph, the frustration index of $G_{-}$is the edge biparticity of $G$, that is, the minimum number of edges whose removal makes the graph bipartite. In Section 6 we illustrate how to use our results in practice with some examples.

Our main results and their proofs show that the weight hierarchies of the incidence matrix code $C$ and its dual code $C^{\perp}$ of a signed graph $G_{\sigma}$ can be computed using the field $\mathbb{Q}$ of rational numbers as the ground field. To compute the GHWs of $C$ and $C^{\perp}$ over a finite field $\mathbb{F}_{q}$ of characteristic $p$, we use the incidence matrix of $G_{\sigma}$ (resp. $G_{+}$) over the field $\mathbb{Q}$ if $p \neq 2$ (resp. $p=2$ ). One can also use the rational numbers to compute the cycles, circuits, and cocircuits of a signed graph, as well as its $r$-th cogirth, frustration index, and $r$-th edge connectivity. In Appendix A we give procedures for Macaulay2 [14 that allow us to obtain this information for graphs with a small number of vertices, see the examples of Section 6. The package Matroids [8] plays an important role here because it computes the circuits and cocircuits of vector matroids over the field of rational numbers; however the problem of computing all circuits of a vector matroid is likely to be NP-hard [21, 38, (cf. [19, p. 76]). The minimum distance of any linear code can be computed using SageMath [30]. For signed simple graphs one can also compute the minimum distance using Proposition 5.6] and the algorithms of [12, 24]. For methods to calculate higher weight enumerators of linear codes see [5] and the references therein.

\section{Matroids AND Linear CODES}

A matroid is a pair $M=(E, \rho)$ where $E$ is a finite set, called the ground set of $M$, and $\rho: 2^{E} \rightarrow \mathbb{N}_{0}:=\{0,1, \ldots\}$ is a function, called the rank function of $M$, satisfying:

$\left(\mathrm{R}_{0}\right) \rho(\emptyset)=0$;

$\left(\mathrm{R}_{1}\right)$ If $X \subseteq E$ and $e \in E$, then $\rho(X) \leq \rho(X \cup\{e\}) \leq \rho(X)+1$;

$\left(\mathrm{R}_{2}\right)$ If $X \subseteq E$ and $Y \subseteq E$, then $\rho(X \cup Y)+\rho(X \cap Y) \leq \rho(X)+\rho(Y)$.

An independent set of a matroid $M$ is subset $X \subseteq E$ such that $\rho(X)=|X|$. In particular the empty set is an independent set. A base is a maximal independent set. A subset of the ground set which is not independent is called dependent and a circuit of $M$ is a minimal dependent set. We denote by $\mathcal{C}_{M}$ the family of all circuits of $M$. The rank of the matroid $M$, denoted $\rho(M)$, is $\rho(E)$. The nullity of $X \subseteq E$, denoted $\eta(X)$, is defined by

$$
\eta(X):=|X|-\rho(X),
$$

and the nullity of $M$, denoted $\eta(M)$, is $\eta(E)$. Let $M=(E, \rho)$ be a matroid. Its dual is the matroid $M^{*}=\left(E, \rho^{*}\right)$ with the same ground set $E$ and rank function given by

$$
\rho^{*}(X):=|X|-\rho(E)+\rho(E \backslash X), \quad X \subseteq E,
$$

see [28, p. 72]. The nullity function of $M^{*}$ is denoted by $\eta^{*}$. One can verify that $\left(M^{*}\right)^{*}=M$.

A family of circuits $\left\{C_{i}\right\}_{i=1}^{r}$ of a matroid $M$ is called non-redundant if $\bigcup_{i \neq j} C_{i} \subsetneq \bigcup_{i=1}^{r} C_{i}$ for $j=1, \ldots, r$ [4, 7]. Let $X$ be a subset of the ground set $E$. The degree or non-redundancy of $X$ is the maximum number of non-redundant circuits contained in $X$, and it is denoted by $\operatorname{deg}(X)$. 
Lemma 2.1. [7, p. 306, Table A.1(6)] Let $M=(E, \rho)$ be a matroid, let $X$ be a subset of $E$, and let $\eta$ be the nullity function of $M$. Then $\operatorname{deg}(X)=\eta(X)$.

Theorem 2.2. [42, Theorem 2] Let $C$ be an $[m, k]$-linear code and let $M^{*}$ be the dual of the vector matroid of $C$. Then, the $r$-th generalized Hamming weight of $C$ is given by

$$
\delta_{r}(C)=\min \left\{|X|: X \subseteq E \text { and } \eta^{*}(X) \geq r\right\} \text { for } 1 \leq r \leq k .
$$

By Lemma 2.1, we can replace the inequality $\eta^{*}(X) \geq r$ by $\eta^{*}(X)=r$. This result suggests how to define the generalized Hamming weights of any matroid $M$.

Definition 2.3. [6, p. 332] Let $M=(E, \rho)$ be a matroid with nullity function $\eta$. The generalized Hamming weights of $M$ are defined as

$$
d_{r}(M):=\min \{|X|: X \subseteq E \text { and } \eta(X)=r\} \text { for } 1 \leq r \leq \eta(E) .
$$

Lemma 2.4. Let $C$ be a linear code of length $m$ and dimension $k$ and let $M$ be its vector matroid. Then $\delta_{r}(C)=d_{r}\left(M^{*}\right)$ for $1 \leq r \leq k$ and $\delta_{r}\left(C^{\perp}\right)=d_{r}(M)$ for $1 \leq r \leq m-k$.

Proof. By Lemma 2.1 and Theorem 2.2, we obtain $\delta_{r}(C)=d_{r}\left(M^{*}\right)$ for $1 \leq r \leq k$. The matroid associated to $C^{\perp}$ is $M^{*}$. Hence $\delta_{r}\left(C^{\perp}\right)=d_{r}(M)$ for $1 \leq r \leq m-k$.

Theorem 2.5. ([1, Corollary 1.3], [20, Proposition 6]) Let $M=(E, \rho)$ be a matroid and let $\eta$ be its nullity function. The following hold.

$$
\begin{aligned}
& d_{r}\left(M^{*}\right)=\min \{|X|: X \subseteq E \text { and } \rho(E \backslash X)=\rho(E)-r\} \text { for } 1 \leq r \leq \rho(E) . \\
& d_{r}(M)=\min \left\{\left|\bigcup_{i=1}^{r} C_{i}\right|:\left\{C_{i}\right\}_{i=1}^{r} \text { are non-redundant circuits of } M\right\} \text { for } 1 \leq r \leq \eta(E) .
\end{aligned}
$$

Proof. According to [44, Theorem 2, p. 35], one has $\rho(E \backslash X)=\rho(E)-\eta^{*}(X)$. Therefore the first equality follows from

$$
d_{r}\left(M^{*}\right)=\min \left\{|X|: X \subseteq E \text { and } \eta^{*}(X)=r\right\} \text { for } 1 \leq r \leq \rho(E) .
$$

On the other hand, recall that by definition of $d_{r}(M)$, one has

$$
d_{r}(M)=\min \{|X|: X \subseteq E \text { and } \eta(X)=r\} \text { for } 1 \leq r \leq \eta(E) .
$$

Therefore, applying Lemma 2.1, the second equality follows.

Corollary 2.6. Let $C$ be an $[m, k]$-linear code and let $M=(E, \rho)$ be the vector matroid of $C$. Then the following equalities hold:

$$
\begin{aligned}
& \delta_{r}(C)=\min \{|X|: X \subseteq E \text { and } \rho(E \backslash X)=\rho(E)-r\} \text { for } 1 \leq r \leq k . \\
& \delta_{r}\left(C^{\perp}\right)=\min \left\{\left|\bigcup_{i=1}^{r} C_{i}\right|:\left\{C_{i}\right\}_{i=1}^{r} \text { are non-redundant circuits of } M\right\} \text { for } 1 \leq r \leq m-k .
\end{aligned}
$$

Proof. By Lemma 2.4, we obtain $\delta_{r}(C)=d_{r}\left(M^{*}\right)$ for $1 \leq r \leq k$ and $\delta_{r}\left(C^{\perp}\right)=d_{r}(M)$ for $1 \leq r \leq m-k$. Thus the result follows from Theorem 2.5.

The number in the right hand side of the second equality of Corollary 2.6 is the $r$-th circuit number of $M$ and is denoted $\gamma_{r}(M)$ [4]. The $r$-th cocircuit number $\beta_{r}(M)$ is defined similarly.

If $C$ is an $[m, k]$-linear code, then $\delta_{1}(C)<\cdots<\delta_{k}(C)$ [18, 42]. The following duality theorem of Wei is a classical result in this area.

Theorem 2.7. (Wei's duality [42, Theorem 3]) Let $C$ be an $[m, k]$-linear code. Then

$$
\left\{\delta_{r}(C) \mid r=1, \ldots, k\right\}=\{1, \ldots, m\} \backslash\left\{m+1-\delta_{r}\left(C^{\perp}\right) \mid r=1, \ldots, m-k\right\} .
$$

This result was generalized by Britz, Johnsen, Mayhew and Shiromoto [6, Theorem 5] from linear codes to arbitrary matroids. 


\section{Generalized Hamming Weights over signed Graphs}

In this section we present our main results on linear codes. To avoid repetitions, we continue to employ the notations and definitions used in Sections 1 and 2 .

A multigraph $G$ consists of a finite set of vertices, $V(G)$, and a finite multiset of edges, $E(G)$. Edges of $G$ are of two types. A link $e=\{v, w\}$, with two distinct endpoints, $v, w$ in $V(G)$ and a loop, $e=\{v, v\}$, with two coincident endpoints. As $E(G)$ is a multiset, multiple edges are allowed. The number of edges of $G$ counted with multiplicity is denoted by $m=|E(G)|$. A multigraph with no loops or multiple edges is called a simple graph or a graph. Let $G$ be a multigraph. A cycle of $G$ is a simple closed path in $G$. A loop is a cycle of length 1, a pair of parallel links is a cycle of length 2 , a triangle is a cycle of length 3 , and so on. A maximal connected subgraph of a graph is called a connected component of the graph.

Theorem 3.1. ([46, Theorem 5.1], [48, Theorem 2.1]) Let $G_{\sigma}$ be a signed graph. Then there exists a matroid $M\left(G_{\sigma}\right)$ on $E(G)$ whose circuits are the balanced cycles and the bowties of $G_{\sigma}$.

The matroid $M\left(G_{\sigma}\right)$ is called the signed-graphic matroid of $G_{\sigma}$. The circuits of $M\left(G_{\sigma}\right)$ are called the circuits of $G_{\sigma}$. If $G_{\sigma}$ is balanced, then $M\left(G_{\sigma}\right)$ is the graphic matroid $M(G)$ of $G$.

Definition 3.2. Let $G_{\sigma}$ be an unbalanced (resp. balanced) signed graph. A cutset of $G_{\sigma}$ is a set of edges whose removal from $G_{\sigma}$ increases the number of balanced connected components (resp. connected components) of $G_{\sigma}$. A cocircuit of $G_{\sigma}$ is a minimal cutset of $G_{\sigma}$. If $G_{\sigma}$ is balanced, a cocircuit of $G_{\sigma}$ is called a cocycle or bond of $G$.

Lemma 3.3. [28, Proposition 2.3.1] If $G_{\sigma}$ is a balanced signed graph, then the cocircuits of $G_{\sigma}$ are the cocircuits of the graphic matroid $M(G)$ of $G$, that is, the circuits of $M(G)^{*}$.

Lemma 3.4. [46, Theorem 5.1(i)] If $G_{\sigma}$ is a connected unbalanced signed graph and $M\left(G_{\sigma}\right)$ is its signed-graphic matroid, then the cocircuits of $G_{\sigma}$ are the cocircuits of $M\left(G_{\sigma}\right)$, that is, the circuits of the dual matroid $M\left(G_{\sigma}\right)^{*}$.

Proof. Let $\rho$ be the rank function of $M\left(G_{\sigma}\right)$. We set $V=V(G)$ and $E=E(G)$. Take a cocircuit $X \subseteq E$ of $G_{\sigma}$. As $G_{\sigma}$ is connected, one has $c_{0}(E)=0$ and $c_{0}(E \backslash X)=1$ if $G_{\sigma}$ is unbalanced, and $c_{0}(E)=1$ and $c_{0}(E \backslash X)=2$ if $G_{\sigma}$ is balanced. Then $c_{0}(E \backslash X)=c_{0}(E)+1$. According to [46, Theorem 5.1(j)], one has

$$
\rho(E)=|V|-c_{0}(E) \text { and } \rho(E \backslash X)=|V|-c_{0}(E \backslash X) .
$$

Therefore $\rho(E \backslash X)=\rho(E)-1$. Since $X$ is a minimal cutset, it follows that $H:=E \backslash X$ is closed, that is, $\rho(H \cup\{e\})=\rho(H)+1=\rho(E)$ for each $e \notin H$. Indeed, from the equality $H \cup\{e\}=E \backslash(X \backslash\{e\})$, and the minimality of $X$, we get $c_{0}(H \cup\{e\})=c_{0}(E)$. Hence, using Eq. (3.1), we obtain $\rho(H \cup\{e\})=\rho(E)=\rho(H)+1$ for each $e \notin H$. As a consequence, $H$ is a maximal set of rank $\rho(E)-1$. Thus, by [44, Lemma 1, p. 38], $H$ is a hyperplane of $M\left(G_{\sigma}\right)$ in the sense of [44], and by [44, Theorem 2, p. 39], $X$ is a cocircuit of $M\left(G_{\sigma}\right)$. Similarly, if $X$ is a cocircuit of $M\left(G_{\sigma}\right)$, it is seen that $X$ is a cocircuit of $G_{\sigma}$.

Lemma 3.5. Let $G_{\sigma}$ be a connected signed graph, let $\rho$ and $\eta$ be the rank and nullity functions of the signed-graphic matroid $M=M\left(G_{\sigma}\right)$ of $G_{\sigma}$. The following hold.

(i) If $1 \leq r \leq \eta(M)$, then $d_{r}(M)$ is equal to the minimum number of edges of $G$ forming a union of $r$ non-redundant balanced cycles and bowties of $G_{\sigma}$.

(ii) If $1 \leq r \leq \rho(M)$ and $G_{\sigma}$ is unbalanced (resp. balanced), then $d_{r}\left(M^{*}\right)$ is the $r$-th cogirth $v_{r}\left(G_{\sigma}\right)$ (resp. $r$-th edge connectivity $\lambda_{r}\left(G_{\sigma}\right)$ ) of $G_{\sigma}$. 
(iii) If $1 \leq r \leq \rho(M)$, then $d_{r}\left(M^{*}\right)$ is equal to the minimum number of edges of $G_{\sigma}$ forming a union of $r$ non-redundant cocircuits of $G_{\sigma}$.

Proof. (i): By Theorem 3.1, the circuits of $M$ are the balanced cycles and the bowties of $G_{\sigma}$. Hence, it suffices to recall the following formula of Theorem 2.5.

$d_{r}(M)=\min \left\{\left|\bigcup_{i=1}^{r} C_{i}\right|:\left\{C_{i}\right\}_{i=1}^{r}\right.$ are non-redundant circuits of $\left.M\right\}$ for $1 \leq r \leq \eta(M)$.

(ii): Let $E$ be the edge set of $G_{\sigma}$, which is the ground set of $M$, and let $V$ be the vertex set of $G_{\sigma}$. According to [46, Theorem 5.1(j)] the rank function of $M\left(G_{\sigma}\right)$ satisfies

$$
\rho(E \backslash X)=|V|-c_{0}(E \backslash X) \text { for } X \subseteq E,
$$

where $c_{0}(E \backslash X)$ is the number of balanced connected components of the signed subgraph $G_{\sigma} \backslash X$ with edge set $E \backslash X$ and vertex set $V$. Therefore, by Theorem 2.5, we obtain

$$
\begin{aligned}
d_{r}\left(M^{*}\right) & =\min \{|X|: X \subseteq E \text { and } \rho(E \backslash X)=\rho(E)-r\} \\
& =\min \left\{|X|: X \subseteq E \text { and }|V|-c_{0}(E \backslash X)=\rho(E)-r\right\}
\end{aligned}
$$

for $1 \leq r \leq \rho(E)$. If $G_{\sigma}$ is unbalanced (resp. balanced), then by making $X=\emptyset$ in Eq. (3.2) we get $\rho(E)=|V|$ (resp. $\rho(E)=|V|-1)$. Therefore

$$
d_{r}\left(M^{*}\right)= \begin{cases}\min \left\{|X|: X \subseteq E \text { and } c_{0}(E \backslash X)=r\right\}=v_{r}\left(G_{\sigma}\right) & \text { if } G_{\sigma} \text { is unbalanced, } \\ \min \{|X|: X \subseteq E \text { and } c(E \backslash X)=r+1\}=\lambda_{r}\left(G_{\sigma}\right) & \text { if } G_{\sigma} \text { is balanced, }\end{cases}
$$

where $c(E \backslash X)$ is the number of connected components of the signed subgraph $G_{\sigma} \backslash X$.

(iii): By Lemmas 3.3 and 3.4, the circuits of the dual matroid $M^{*}$ of $M$ are the cocircuits of the signed graph $G_{\sigma}$, and by Theorem 2.5 we get

$d_{r}\left(M^{*}\right)=\min \left\{\left|\bigcup_{i=1}^{r} C_{i}^{*}\right|:\left\{C_{i}^{*}\right\}_{i=1}^{r}\right.$ are non-redundant circuits of $\left.M^{*}\right\}$ for $1 \leq r \leq \eta^{*}(E)$.

Hence, the required equality follows noticing that $\eta^{*}(E)=\rho(E)$.

Definition 3.6. Let $G_{\sigma}$ be a signed graph with $s$ vertices $t_{1}, \ldots, t_{s}$ and $m$ edges, let $K$ be a field, and let $\mathbf{e}_{i}$ be the $i$-th unit vector in $K^{s}$. The incidence matrix of $G_{\sigma}$ over the field $K$ is the $s \times m$ matrix $A$ whose column vectors are given by:

(i) $\mathbf{e}_{i}-\mathbf{e}_{j}$ (resp. $\mathbf{e}_{i}+\mathbf{e}_{j}$ ) if $e=\left\{t_{i}, t_{j}\right\}$ is a link with $\sigma(e)=+($ resp. $\sigma(e)=-$ );

(ii) $\mathbf{0}\left(\right.$ resp. $\left.2 \mathbf{e}_{i}\right)$ if $e=\left\{t_{i}, t_{i}\right\}$ is a loop with $\sigma(e)=+$ (resp. $\sigma(e)=-$ ).

Note that the columns of $A$ are defined up to sign, so one can pick $\mathbf{e}_{i}-\mathbf{e}_{j}$ or $\mathbf{e}_{j}-\mathbf{e}_{i}$ if $e=\left\{t_{i}, t_{j}\right\}$ is a link with $\sigma(e)=+$. To avoid ambiguity we could normalize and pick $e_{i}-e_{j}$ if $i>j$. The order of the columns of $A$ and the choice of sign have no significance for the invariants of linear codes, signed graphs, and Stanley-Reisner ideals that we want to study.

If $G$ is a multigraph with vertices $t_{1}, \ldots, t_{s}$, the incidence matrix of $G$ over a field $K$ is the incidence matrix of the negative signed graph $G_{-}$, that is, the matrix whose columns are all vectors $\mathbf{e}_{i}+\mathbf{e}_{j}$ such that $\left\{t_{i}, t_{j}\right\}$ is an edge of $G$. A digraph $\mathcal{D}$ consists of a multigraph $G$ with vertices $t_{1}, \ldots, t_{s}$ where all edges of $G$ are directed from one vertex to another. The edges or arrows of $\mathcal{D}$ are ordered pairs of vertices $\left(t_{i}, t_{j}\right)$ with $e=\left\{t_{i}, t_{j}\right\}$ an edge of $G$, where $\left(t_{i}, t_{j}\right)$ represents the edge $e$ directed from $t_{i}$ to $t_{j}$. The incidence matrix of $\mathcal{D}$ over a field $K$ is the incidence matrix of the positive signed graph $G_{+}$, that is, the matrix whose columns are all vectors $\mathbf{e}_{i}-\mathbf{e}_{j}$ such that $\left(t_{i}, t_{j}\right)$ is an edge of $\mathcal{D}$.

Theorem 3.7. [46, Theorems 8B.1, 8B.2] Let $G_{\sigma}$ be a signed graph and let $A$ be its incidence matrix over a field of characteristic $p$. The following hold. 
(a) If $p \neq 2$, then the vector matroid $M[A]$ of $A$ is the signed-graphic matroid $M\left(G_{\sigma}\right)$.

(b) If $p=2$, then $M[A]$ is the graphic matroid $M(G)$ of $G$.

Proposition 3.8. Let $G_{\sigma}$ be a signed graph with s vertices, c connected components, $c_{0}$ balanced connected components, and let $A$ be its incidence matrix over a field $K$. Then

$$
\operatorname{rank}(A)= \begin{cases}s-c_{0} & \text { if } \operatorname{char}(K) \neq 2, \\ s-c & \text { if } \operatorname{char}(K)=2 \text { or } G_{\sigma} \text { is balanced } .\end{cases}
$$

Proof. Assume char $(K) \neq 2$. By Theorem [3.7(a), the signed graphic matroid $M\left(G_{\sigma}\right)$ is the vector matroid $M[A]$. According to [46, Theorem 5.1(j)], the rank of $M\left(G_{\sigma}\right)$ is $s-c_{0}$. Thus in this case $\operatorname{rank}(A)=s-c_{0}$. Assume $\operatorname{char}(K)=2$. By Theorem 3.7(b), the graphic matroid $M(G)$ is the vector matroid $M[A]$. If $G$ is connected, then the bases of the matroid $M(G)$ are the spanning trees of $G$ [44, p. 28], and $\operatorname{rank}(A)=s-1$. As a consequence, if $G$ has $c$ components, one has $\operatorname{rank}(A)=s-c$. If $G_{\sigma}$ is balanced, then $c=c_{0}$, and by the previous two cases $\operatorname{rank}(A)=s-c$, regardless of the characteristic of the field $K$.

Corollary 3.9. Let $\mathcal{D}$ be a digraph with $s$ vertices and $c$ connected components, and let $A$ be its incidence matrix over a field $K$. Then, $\operatorname{rank}(A)=s-c$.

Proof. Let $G$ be the underlying unoriented simple graph of $\mathcal{D}$. Consider the positive signed graph $G_{+}$. Note that $G_{+}$is balanced. Since $\mathcal{D}$ and $G_{+}$have the same incidence matrix, the result follows from Proposition 3.8 .

Definition 3.10. The incidence matrix code of a signed graph $G_{\sigma}$ (resp. multigraph $G$, digraph $\mathcal{D}$ ), over a finite field $\mathbb{F}_{q}$, is the linear code $C$ generated by the rows of the incidence matrix of the signed graph $G_{\sigma}$ (resp. multigraph $G$, digraph $\mathcal{D}$ ).

Corollary 3.11. Let $G_{\sigma}$ be a connected signed graph with $s$ vertices and $m$ edges, and let $C$ be the incidence matrix code of $G_{\sigma}$ over a finite field of characteristic $p$. Then

(a) $C$ (resp. $\left.C^{\perp}\right)$ is an $[m, s]($ resp. $[m, m-s])$ linear code if $p \neq 2$ and $G_{\sigma}$ is unbalanced.

(b) $C\left(\right.$ resp. $\left.C^{\perp}\right)$ is an $[m, s-1]$ (resp. $\left.[m, m-s+1]\right)$ linear code if $p=2$ or $G_{\sigma}$ is balanced.

Proof. This follows from Proposition 3.8 noticing that $\operatorname{dim}(C)+\operatorname{dim}\left(C^{\perp}\right)=m$.

Definition 3.12. Let $G$ be a multigraph. A bowtie of $G$ is the union of two odd cycles which meet at a single vertex or the union of two vertex-disjoint odd cycles and a simple path which meets one cycle at each end and is otherwise disjoint from them.

Corollary 3.13. Let $G$ be a multigraph and let $G_{+}$and $G_{-}$be the positive and negative signed graphs, respectively. The following hold.

(a) The circuits of the signed-graphic matroid $M\left(G_{+}\right)$are the cycles of $G$, that is, $M\left(G_{+}\right)$ is the graphic matroid $M(G)$ of $G$.

(b) The signed-graphic matroid $M\left(G_{+}\right)$is the vector matroid, over any field $K$, of the incidence matrix of $G_{+}$whose columns are of the form $\mathbf{e}_{i}-\mathbf{e}_{j}$.

(c) The balanced (resp. unbalanced) cycles of $G_{-}$are the even (resp. odd) cycles of $G$. A circuit of $M\left(G_{-}\right)$is either an even cycle or a bowtie of $G$.

(d) If $G_{\sigma}$ is a balanced signed graph, then $M\left(G_{\sigma}\right)$ is the graphic matroid $M(G)$ of $G$.

Proof. (a): There are no unbalanced cycles of $G_{+}$. Hence, by Theorem 3.1, the circuits of $M\left(G_{+}\right)$are the cycles of $G$. 
(b): Let $p$ be the characteristic of the field $K$. If $p \neq 2$, by Theorem 3.7, $M\left(G_{+}\right)$is the vector matroid of the incidence matrix $A$ of $G_{+}$and the columns of this matrix have the required form. If $p=2$, the graphic matroid $M(G)$ of $G$ is the vector matroid $M[A]$ of the incidence matrix $A$ of $G$ [44, Theorem 3, p. 149]. By part (a), $M\left(G_{+}\right)$is the graphic matroid of $G$. Thus $M\left(G_{+}\right)$is the vector matroid of $A$. The columns of $A$ have the required form because in this case $1=-1$.

(c), (d): These follow readily from Theorem 3.1.

Remark 3.14. Let $G$ be a multigraph and let $A$ be the incidence matrix of $G_{+}$over the field $K=\mathbb{Q}$ of rational numbers. Since $M[A]$ is the graphic matroid of $G$, to compute all cycles of $G$ one can use Macaulay2 14 and the package Matroids [8].

Corollary 3.15. [33, 39, 46] If $A$ is the incidence matrix of a multigraph $G$ over a field of $\operatorname{char}(K) \neq 2$, then the circuits of the vector matroid $M[A]$ are the even cycles and bowties of $G$.

Proof. It follows from Theorem 3.7(a) and Corollary 3.13)(c) by considering $G_{-}$.

Our main results on linear codes are the following. First, we give graph theoretical formulas for the GHWs for the incidence matrix code of a signed graph.

Theorem 3.16. Let $C$ be the incidence matrix code of a connected signed graph $G_{\sigma}$ with $s$ vertices, $r$-th cogirth $v_{r}\left(G_{\sigma}\right)$, $r$-th edge connectivity $\lambda_{r}(G)$, over a finite field $K$ of char $(K)=p$. Then, the $r$-th generalized Hamming weight of $C$ is given by

$$
\delta_{r}(C)= \begin{cases}v_{r}\left(G_{\sigma}\right) & \text { if } p \neq 2, G_{\sigma} \text { is unbalanced and } 1 \leq r \leq s, \\ \lambda_{r}(G) & \text { if } p=2 \text { and } 1 \leq r \leq s-1, \\ \lambda_{r}(G) & \text { if } G_{\sigma} \text { is balanced and } 1 \leq r \leq s-1 .\end{cases}
$$

Proof. Let $A$ be the incidence matrix of $G_{\sigma}$ and let $\rho$ be the rank function of the vector matroid $M=M[A]$. According to Proposition 3.8, $\rho(M)=s$ if $p \neq 2$ and $G_{\sigma}$ is unbalanced, and $\rho(M)=s-1$ if $p=2$ or $G_{\sigma}$ is balanced.

Assume that $p \neq 2$. By Theorem [3.7(a), the signed-graphic matroid $M\left(G_{\sigma}\right)$ is the vector matroid $M=M[A]$. Hence, using Lemmas 2.4 and 3.5(ii), one has

$$
\delta_{r}(C)=d_{r}\left(M^{*}\right)= \begin{cases}v_{r}\left(G_{\sigma}\right) & \text { if } G_{\sigma} \text { is unbalanced and } 1 \leq r \leq s, \\ \lambda_{r}(G) & \text { if } G_{\sigma} \text { is balanced and } 1 \leq r \leq s-1 .\end{cases}
$$

Assume that $p=2$. By Theorem $3.7(\mathrm{~b}), M=M[A]$ is the graphic matroid $M(G)$ and, by Corollary $3.13(\mathrm{a}), M\left(G_{+}\right)$is also the graphic matroid $M(G)$. As $M\left(G_{+}\right)$is balanced, by Lemmas 2.4 and 3.5 (ii), we get $\delta_{r}(C)=d_{r}\left(M^{*}\right)=\lambda_{r}\left(G_{+}\right)=\lambda_{r}(G)$.

Let $G$ be a multigraph. The $r$-th cogirth $v_{r}\left(G_{-}\right)$of $G_{-}$is the minimum number of edges whose removal results in a multigraph with $r$ bipartite connected components. If $r=1, v_{1}\left(G_{-}\right)$ is denoted $v\left(G_{-}\right)$. For simple graphs, the following combinatorial formulas for the generalized Hamming weights were shown in [26].

Corollary 3.17. Let $C$ be the incidence matrix code of a connected multigraph $G$ with s vertices over a finite field $K$ of $\operatorname{char}(K)=p$. Then

$$
\delta_{r}(C)= \begin{cases}v_{r}\left(G_{-}\right) & \text {if } p \neq 2, G \text { is non-bipartite and } 1 \leq r \leq s \\ \lambda_{r}(G) & \text { if } p=2 \text { and } 1 \leq r \leq s-1 \\ \lambda_{r}(G) & \text { if } G \text { is bipartite and } 1 \leq r \leq s-1 .\end{cases}
$$


Proof. It follows from Theorem 3.16 by considering the negative signed graph $G_{-}$and noticing that $G_{-}$is balanced if and only if $G$ is bipartite.

The next result shows combinatorial formulas for the minimum distance of the incidence matrix code of a signed graph [28, Proposition 9.2.4].

Corollary 3.18. Let $C$ be the incidence matrix code of a connected signed graph $G_{\sigma}$ with $s$ vertices, cogirth $v\left(G_{\sigma}\right)$, edge connectivity $\lambda\left(G_{\sigma}\right)$, over a finite field $K$ of $\operatorname{char}(K)=p$. Then, the minimum distance $\delta(C)$ of $C$ is given by

$$
\delta(C)= \begin{cases}v\left(G_{\sigma}\right) & \text { if } p \neq 2, G_{\sigma} \text { is unbalanced and } 1 \leq r \leq s \\ \lambda\left(G_{\sigma}\right) & \text { if } p=2 \text { and } 1 \leq r \leq s-1 \\ \lambda\left(G_{\sigma}\right) & \text { if } G_{\sigma} \text { is balanced and } 1 \leq r \leq s-1\end{cases}
$$

Proof. It follows by making $r=1$ in Theorem 3.16 .

Our next result gives graph theoretical formulas for the generalized Hamming weights of the dual code of the incidence matrix code of a signed graph.

Theorem 3.19. Let $G_{\sigma}$ be a connected signed graph with $s$ vertices and $m$ edges, and let $C$ be the incidence matrix code of $G_{\sigma}$ over a finite field $K$ of characteristic $p$. The following hold.

(a) If $p=2$ or $G_{\sigma}$ is balanced, and $1 \leq r \leq m-s+1$ (resp. $\left.1 \leq r \leq s-1\right)$, then $\delta_{r}\left(C^{\perp}\right)$ (resp. $\delta_{r}(C)$ ) is the minimum number of edges of $G$ forming a union of $r$ non-redundant cycles (resp. cocycles) of $G$.

(b) If $p \neq 2$ and $1 \leq r \leq m-s$ (resp. $1 \leq r \leq s)$, then $\delta_{r}\left(C^{\perp}\right)\left(\right.$ resp. $\left.\delta_{r}(C)\right)$ is the minimum number of edges of $G$ forming a union of $r$ non-redundant balanced cycles and bowties (resp. cocircuits) of $G_{\sigma}$.

Proof. (a): Assume $p=2$ and $1 \leq r \leq m-s+1$. Let $A$ be the incidence matrix of $G_{\sigma}$. By Theorem 3.7(b), the vector matroid $M=M[A]$ is the graphic matroid $M(G)$. Thus the circuits of $M[A]$ are the cycles of $G$. Therefore, by Corollary 2.6, we get

$$
\delta_{r}\left(C^{\perp}\right)=\min \left\{\left|\bigcup_{i=1}^{r} C_{i}\right|:\left\{C_{i}\right\}_{i=1}^{r} \text { are non-redundant cycles of } G\right\} .
$$

Assume $p=2$ and $1 \leq r \leq s-1$. By the previous part, $M=M[A]$ is the graphic matroid $M(G)$. The circuits of $M^{*}=M[A]^{*}$ are the cocycles of $G$ [44, p. 41], that is, these are edge sets $X$ whose removal from $G$ increases the number of connected components of $G$ and are minimal with respect to this property. The dual matroid $M^{*}$ of $M$ is the vector matroid of $C^{\perp}$ [44, p. 141]. Therefore, by Corollary 2.6 and the equality $C=\left(C^{\perp}\right)^{\perp}$, we get

$$
\begin{aligned}
\delta_{r}(C) & =\min \left\{\left|\bigcup_{i=1}^{r} C_{i}^{*}\right|:\left\{C_{i}^{*}\right\}_{i=1}^{r} \text { are non-redundant circuits of } M^{*}\right\} \\
& =\min \left\{\left|\bigcup_{i=1}^{r} C_{i}^{*}\right|:\left\{C_{i}^{*}\right\}_{i=1}^{r} \text { are non-redundant cocycles of } G\right\} .
\end{aligned}
$$

Assume that $G_{\sigma}$ is balanced. If $p=2$, the formulas for $\delta_{r}\left(C^{\perp}\right)$ and $\delta_{r}(C)$ follow from the two previous cases. Assume $p \neq 2$. By Theorem 3.7(a), the vector matroid $M[A]$ is the signedgraphic matroid $M\left(G_{\sigma}\right)$ and, by Corollary $3.13(\mathrm{~d}), M\left(G_{\sigma}\right)$ is the graphic matroid $M(G)$. Hence, we can proceed as in the previous cases.

(b): Assume $1 \leq r \leq m-s$. Let $A$ be the incidence matrix of $G_{\sigma}$. By Theorem 3.1, the circuits of $M\left(G_{\sigma}\right)$ are the balanced cycles and bowties of $G_{\sigma}$. As $p \neq 2$, by Theorem 3.7(a), the vector matroid $M=M[A]$ is $M\left(G_{\sigma}\right)$. Therefore the circuits of $M[A]$ are the balanced cycles and bowties of $G_{\sigma}$, and by Corollary 2.6 one has

$$
\delta_{r}\left(C^{\perp}\right)=\min \left\{\left|\bigcup_{i=1}^{r} C_{i}\right|:\left\{C_{i}\right\}_{i=1}^{r} \text { are non-redundant circuits of } M\right\} .
$$


Assume $1 \leq r \leq s$. As $M=M[A]$ is the signed-graphic matroid $M\left(G_{\sigma}\right)$, the circuits of $M^{*}=M[A]^{*}$ are the cocircuits of $G_{\sigma}$ by Lemmas 3.3 and 3.4. The dual matroid $M^{*}$ of $M$ is the vector matroid of $C^{\perp}$ [44, p. 141]. Hence, by Corollary 2.6 and noticing $C=\left(C^{\perp}\right)^{\perp}$, we get

$$
\begin{aligned}
\delta_{r}(C) & =\min \left\{\left|\bigcup_{i=1}^{r} C_{i}^{*}\right|:\left\{C_{i}^{*}\right\}_{i=1}^{r} \text { are non-redundant circuits of } M^{*}\right\} \\
& =\min \left\{\left|\bigcup_{i=1}^{r} C_{i}^{*}\right|:\left\{C_{i}^{*}\right\}_{i=1}^{r} \text { are non-redundant cocircuits of } G_{\sigma}\right\} .
\end{aligned}
$$

This completes the proof of part (b).

Corollary 3.20. Let $C$ be the incidence matrix code, over a finite field $K=\mathbb{F}_{q}$, of a connected digraph $\mathcal{D}$ with $s$ vertices and $m$ edges, and let $G$ be its underlying multigraph. Then

(a) $\delta_{r}(C)=\lambda_{r}(G)$ for $1 \leq r \leq s-1$.

(b) If $1 \leq r \leq m-s+1$ (resp. $1 \leq r \leq s-1)$, then $\delta_{r}\left(C^{\perp}\right)$ (resp. $\left.\delta_{r}(C)\right)$ is the minimum number of edges of $G$ forming a union of $r$ non-redundant cycles (resp. cocycles) of $G$.

Proof. Parts (a) and (b) follow from Theorems 3.16 and 3.19, respectively, by considering the positive signed graph $G_{+}$and noticing that this is a balanced signed graph.

Corollary 3.21. Let $G$ be a connected multigraph with $s$ vertices and $m$ edges, and let $C$ be its incidence matrix code over a finite field $K=\mathbb{F}_{q}$ of characteristic $p$. The following hold.

(a) If $p=2$ or $G$ is bipartite, and $1 \leq r \leq m-s+1$ (resp. $1 \leq r \leq s-1)$, then $\delta_{r}\left(C^{\perp}\right)$ (resp. $\left.\delta_{r}(C)\right)$ is the minimum number of edges of $G$ forming a union of $r$ non-redundant cycles (resp. cocycles) of $G$.

(b) If $p \neq 2$ and $1 \leq r \leq m-s$, then $\delta_{r}\left(C^{\perp}\right)$ is the minimum number of edges of $G$ forming a union of $r$ non-redundant even cycles and bowties of $G$.

Proof. (a): This follows from Theorem 3.19(a) noticing that, if $G$ is bipartite, then the circuits of $M\left(G_{+}\right)$and $M\left(G_{-}\right)$are the cycles of $G$.

(b): This part follows from Corollary 3.13(c) and Theorem 3.19(b), by considering the negative signed graph $G_{-}$.

\section{The REgUlarity OF THE IDEAL OF CIRCUITS}

Let $M$ be a matroid on $E=\{1, \ldots, m\}$, let $\Delta$ be its independence complex, that is, the faces of $\Delta$ are the independent sets of $M$, and let $R=\mathbb{Q}\left[x_{1}, \ldots, x_{m}\right]=\bigoplus_{d=0}^{\infty} R_{d}$ be a polynomial ring with the standard grading over the field of rational numbers. It is convenient also to think of $E$ as the set of variables $\left\{x_{1}, \ldots, x_{m}\right\}$. The Stanley-Reisner ideal $I_{\Delta}$ of $\Delta$, in the sense of [36], is the edge ideal $I\left(\mathcal{C}_{M}\right)$ of the clutter of circuits $\mathcal{C}_{M}$ of $M$, that is, $I_{\Delta}$ is the ideal of circuits of $M$ generated by all squarefree monomials $\prod_{i \in X} x_{i}$ such that $X$ is a circuit of $M$.

The simplicial complex $\Delta$ is pure shellable, in particular the ideal $I_{\Delta}$ is Cohen-Macaulay, and the graded Betti numbers of the Stanley-Reisner ring $K[\Delta]=R / I_{\Delta}$ are the same if we replace $\mathbb{Q}$ by any other field (see [19, Remark 1, p. 78] and the references therein).

Definition 4.1. Let $I \subset R$ be a graded ideal and let $\mathbf{F}$ be the minimal graded free resolution of $R / I$ as an $R$-module:

$$
\mathbf{F}: \quad 0 \rightarrow \bigoplus_{j} R(-j)^{\beta_{g, j}} \rightarrow \cdots \rightarrow \bigoplus_{j} R(-j)^{\beta_{1, j}} \rightarrow R \rightarrow R / I \rightarrow 0 .
$$

The $(i, j)$-th graded Betti number of $R / I$, denoted $\beta_{i, j}(R / I)$, is $\beta_{i, j}$, the integer $j$ is a shift of the resolution, $g$ is the projective dimension of $R / I$, and the regularity of $R / I$ is

$$
\operatorname{reg}(R / I):=\max \left\{j-i \mid \beta_{i, j} \neq 0\right\} \text {. }
$$


If $R / I$ is Cohen-Macaulay (i.e. $g=\operatorname{dim}(R)-\operatorname{dim}(R / I)$ ) and there is a unique $j$ such that $\beta_{g, j} \neq 0$, then the ring $R / I$ is called level.

An excellent reference for the regularity of graded ideals and Betti numbers is the book of Eisenbud [10]. The shifts and the Betti numbers of the Stanley-Reisner ring of the independence complex $\Delta$ of a matroid $M$ were determined by Johnsen and Verdure [19].

The following result shows that one can read the generalized Hamming weights of a matroid $M$ from the minimal graded free resolution of the ideal of circuits of $M$.

Theorem 4.2. [19, Theorem 2] Let $M$ be a matroid, let $R / I_{\Delta}$ be the Stanley-Reisner ring of the independence complex $\Delta$ of $M$, and let $\beta_{r, j}(M)$ denote the $(r, j)$-th graded Betti number of $R / I_{\Delta}$. Then the generalized Hamming weights of $M$ are given by

$$
d_{r}(M)=\min \left\{j: \beta_{r, j}(M) \neq 0\right\} \text { for } 1 \leq r \leq \eta(M) .
$$

Corollary 4.3. Let $C$ be an $[m, k]$-linear code and let $R / I_{\Delta}$ be the Stanley-Reisner ring of the independence complex of the matroid of $C$. Then

$$
\delta_{r}\left(C^{\perp}\right)=\min \left\{j: \beta_{r, j}\left(R / I_{\Delta}\right) \neq 0\right\} \text { for } 1 \leq r \leq m-k .
$$

Proof. It follows from Lemma 2.4 and Theorem 4.2 .

The following notion of a non-degenerate code will play a role here.

Definition 4.4. If $C \subseteq K^{m}$ is a linear code and $\pi_{i}$ is the $i$-th projection map

$$
\pi_{i}: C \rightarrow K, \quad\left(v_{1}, \ldots, v_{m}\right) \mapsto v_{i},
$$

for $i=1, \ldots, m$, we say that $C$ is degenerate if for some $i$ the image of $\pi_{i}$ is zero, otherwise we say that $C$ is non-degenerate.

Remark 4.5. If $C \subseteq K^{m}$ is a non-degenerate linear code, then $\delta_{k}(C)=m$, where $k$ is the dimension of $C$. If all columns of a generator matrix of $C$ are non-zero, then $\pi_{i} \neq 0$ for $i=1, \ldots, m$ and $C$ is non-degenerate.

Lemma 4.6. Let $M$ be the matroid on $E$ of a linear code $C$ and let $\Delta\left(\right.$ resp. $\left.\Delta^{*}\right)$ be the independence complex of $M$ (resp. $\left.M^{*}\right)$. The following hold.

(a) If $r=\operatorname{dim}\left(C^{\perp}\right)$, then $\operatorname{reg}\left(R / I_{\Delta}\right)=\delta_{r}\left(C^{\perp}\right)-\operatorname{dim}\left(C^{\perp}\right)$.

(b) If $r=\operatorname{dim}(C)$, then $\operatorname{reg}\left(R / I_{\Delta^{*}}\right)=\delta_{r}(C)-\operatorname{dim}(C)$.

(c) If $C^{\perp}$ is non-degenerate, then $\operatorname{reg}\left(R / I_{\Delta}\right)=\operatorname{dim}(C)$.

(d) If $C$ is non-degenerate, then $\operatorname{reg}\left(R / I_{\Delta^{*}}\right)=\operatorname{dim}\left(C^{\perp}\right)$.

Proof. By [41, Corollary 6.3.5], the Stanley-Reisner ring $K[\Delta]:=R / I_{\Delta}$ has Krull dimension $\operatorname{dim}(\Delta)+1$. As $\operatorname{dim}(\Delta)$ is $\rho(M)-1$ and $\operatorname{dim}(R)=|E|$, one has $|E|-\operatorname{ht}\left(I_{\Delta}\right)=\rho(M)$, where $\operatorname{ht}\left(I_{\Delta}\right)$ is the height of the ideal $I_{\Delta}$. Therefore

$$
\begin{aligned}
\eta(M) & =|E|-\rho(M)=\operatorname{ht}\left(I_{\Delta}\right) \\
& =|E|-\operatorname{dim}(C)=\operatorname{dim}\left(C^{\perp}\right)=\rho\left(M^{*}\right) .
\end{aligned}
$$

Thus $\eta(M)=\operatorname{ht}\left(I_{\Delta}\right)=\operatorname{dim}\left(C^{\perp}\right)=\rho\left(M^{*}\right)$. The independence complex $\Delta$ is pure shellable [19, Remark 1, p. 78]. Hence $I_{\Delta}$ is Cohen-Macaulay, that is, $\operatorname{ht}\left(I_{\Delta}\right)$ is equal to $\operatorname{pd}_{R}\left(R / I_{\Delta}\right)$, the projective dimension of $R / I_{\Delta}$. Let $\beta_{r, j}$ be the $(r, j)$-th graded Betti number of $R / I_{\Delta}$, with 
$r=\eta(M)=\operatorname{pd}_{R}\left(R / I_{\Delta}\right)$. According to [36, Theorem 3.4], the ring $R / I_{\Delta}$ is level. Therefore, by making $r=\operatorname{dim}\left(C^{\perp}\right)$ in Corollary 4.3 , we get

$$
\begin{aligned}
\operatorname{reg}\left(R / I_{\Delta}\right) & =\max \left\{j-r \mid \beta_{r, j} \neq 0\right\}=\min \left\{j-r \mid \beta_{r, j} \neq 0\right\} \\
& =\min \left\{j \mid \beta_{r, j} \neq 0\right\}-r=\delta_{r}\left(C^{\perp}\right)-\operatorname{dim}\left(C^{\perp}\right) .
\end{aligned}
$$

Thus the equality of (a) holds. The equality of (b) follows from (a) using duality. Parts (c) and (d) follow readily from Remark 4.5.

Theorem 4.7. Let $G_{\sigma}$ be a signed graph without loops with $s$ vertices, $m$ edges, $c$ connected components, $c_{0}$ balanced components, let $M$ be the matroid on $E$ of the incidence matrix code $C$ of $G_{\sigma}$, over a finite field of characteristic $p$, and let $\Delta\left(\right.$ resp. $\left.\Delta^{*}\right)$ be the independence complex of $M$ (resp. $\left.M^{*}\right)$. The following hold.

$$
\operatorname{reg}(R / I)= \begin{cases}m-s+c_{0} & \text { if } I=I_{\Delta^{*}}, p \neq 2, \\ m-s+c & \text { if } I=I_{\Delta^{*}}, p=2 \text { or } G_{\sigma} \text { is balanced, } \\ s-c_{0} & \text { if } I=I_{\Delta}, p \neq 2, \text { and any } i \in E \text { is in some circuit of } M, \\ s-c & \text { if } I=I_{\Delta}, p=2 \text { or } G_{\sigma} \text { is balanced, and } G \text { has no bridges. }\end{cases}
$$

Proof. Let $A$ be the incidence matrix of $G_{\sigma}$. As $G_{\sigma}$ has no loops, all columns of $A$ are non-zero, that is, $C$ is non-degenerate. Hence, the first two formulas follow at once from Proposition 3.8 , Lemma 4.6(d), and the equality $\operatorname{dim}\left(C^{\perp}\right)=m-\operatorname{dim}(C)$.

Assume that $p \neq 2$ and suppose any $i \in E$ is in some circuit of $M$. Let $H$ be the parity check matrix of $C$ whose rows correspond to the circuits of $C$ (see the discussion below). The matrix $H$ is a generator matrix for $C^{\perp}$ and $M^{*}$ is the vector matroid $M[H]$. Let $\mathbf{v}_{1}, \ldots, \mathbf{v}_{m}$ be the column vectors of $A$. Take any $i \in E$, then $i$ is in some circuit $X \subseteq E$ of $M$. Then $\sum_{j \in X} \lambda_{j} \mathbf{v}_{j}=0$, where $\lambda_{j} \neq 0$ for $j \in X$. Setting $\lambda_{j}=0$ for $j \in E \backslash X$, we get that $\lambda=\left(\lambda_{1}, \ldots, \lambda_{m}\right)$ is a row of $H$ and $\lambda_{i} \neq 0$. Thus the $i$-th column of $H$ is non-zero for $i=1, \ldots, m$, that is, $C^{\perp}$ is non-degenerate. Therefore, the third formula follows from Proposition 3.8 and Lemma 4.6(c).

Assume that $p=2$ or $G_{\sigma}$ is balanced, and suppose $G$ has no bridges. Then the vector matroid $M$ is the graphic matroid of $G$. As $G$ has no bridges, i.e., any edge belongs to a cycle, one has that every edge is in some circuit of $M$. Hence, by the previous part, $C^{\perp}$ is non-degenerate. Hence, by Proposition 3.8 and Lemma 4.6(c), the fourth equality follows.

\section{An ALGEBRAiC FORMUla FOR THE FRUSTRATION INDEX}

Let $G_{\sigma}$ be a connected signed simple graph with $s$ vertices, $m$ edges, frustration index $\varphi\left(G_{\sigma}\right)$, and let $V\left(G_{\sigma}\right)=\left\{t_{1}, \ldots, t_{s}\right\}$ be its vertex set. For use below, $\mathbb{X}$ will denote the set of projective points in the projective space $\mathbb{P}^{s-1}$ defined by the column vectors of the incidence matrix of $G_{\sigma}$ over a field $K$ of $\operatorname{char}(K) \neq 2$. Consider a polynomial ring $S=K\left[t_{1}, \ldots, t_{s}\right]=\bigoplus_{d=0}^{\infty} S_{d}$ over a field $K$ with the standard grading. Given a homogeneous polynomial $h$ in $S$, that is, $h \in S_{d}$ for some $d$, we denote the set of zeros of $h$ in $\mathbb{X}$ by $V_{\mathbb{X}}(h)$. The vanishing ideal of $\mathbb{X}$, denoted $I(\mathbb{X})$, is the ideal of $S$ generated by the homogeneous polynomials that vanish at all points of $\mathbb{X}$.

The following characterization of balanced signed graphs is due to Harary [15]. For other characterizations of this property see [46] and the references therein.

Theorem 5.1. ([15, Theorem 3], [46, Proposition 2.1]) A signed simple graph is balanced if and only if its vertex set can be partitioned into two disjoint classes (possible empty), such that an edge is negative if and only if its two endpoints belong to distinct classes. 
Lemma 5.2. Let $G_{\sigma}$ be a connected signed simple graph over a field $K$ of char $(K) \neq 2$. Then

$$
\varphi\left(G_{\sigma}\right)=\min \left\{\left|\mathbb{X} \backslash V_{\mathbb{X}}(h)\right|: h=a_{1} t_{1}+\cdots+a_{s} t_{s}, a_{i} \in\{ \pm 1\} \text { for all } i\right\} .
$$

Proof. Let $\mathbf{v}_{1}, \ldots, \mathbf{v}_{m}$ be the column vectors of the incidence matrix of $G_{\sigma}$. We set $r=\varphi\left(G_{\sigma}\right)$ and let $r_{0}$ be the right hand side of Eq. (5.1). If $G_{\sigma}$ is balanced, using Theorem 5.1, it is not hard to see that there is a linear polynomial $h=a_{1} t_{1}+\cdots+a_{s} t_{s}, a_{i} \in\{ \pm 1\}$ for all $i$, such that $h\left(\mathbf{v}_{i}\right)=0$ for all $i$, that is, $r_{0}=0$ and $\varphi\left(G_{\sigma}\right)=r_{0}$ (see the discussion below). Thus we may assume that $G_{\sigma}$ is not balanced. Pick a minimum set of edges $e_{1}, \ldots, e_{r}$ such that the signed subgraph $H_{\sigma}=G_{\sigma} \backslash\left\{e_{1}, \ldots, e_{r}\right\}$ is balanced. We may assume that $\left\{e_{1}, \ldots, e_{r}, \ldots, e_{m}\right\}$ is the set of edges of $G_{\sigma}$ and that $e_{i}$ corresponds to $\mathbf{v}_{i}$ for $i=1, \ldots, m$. We first show the inequality $r \geq r_{0}$. Note that $V\left(H_{\sigma}\right)=V\left(G_{\sigma}\right)$. According to Theorem 5.1, the vertex set of $H_{\sigma}$ can be partitioned into two disjoint classes $V_{1}$ and $V_{2}$ (possible empty) is such a way that an edge of $H_{\sigma}$ is negative if and only if its two endpoints belong to distinct classes. We set

$$
h:=\sum_{t_{i} \in V_{1}} t_{i}-\sum_{t_{i} \in V_{2}} t_{i} .
$$

To show the inequality $r \geq r_{0}$ it suffices to show the equality $V_{\mathbb{X}}(h)=\left\{\mathbf{v}_{i}\right\}_{i=r+1}^{m}$ because this equality implies $r=\left|\mathbb{X} \backslash V_{\mathbb{X}}(h)\right|$, and consequently $r=\varphi\left(G_{\sigma}\right) \geq r_{0}$.

Case (I): $V_{2}=\emptyset$. Therefore, $\sigma(e)=+$ for $e \in E\left(H_{\sigma}\right)$. As $h=\sum_{i=1}^{s} t_{i}$, one has the inclusion $\left\{\mathbf{v}_{i}\right\}_{i=r+1}^{m} \subseteq V_{\mathbb{X}}(h)$. We claim that $\sigma\left(e_{i}\right)=-$ for $i=1, \ldots, r$. If $\sigma\left(e_{i}\right)=+$ for some $1 \leq i \leq r$, then $G_{\sigma} \backslash\left\{e_{1}, \ldots, e_{i-1}, e_{i+1}, \ldots, e_{r}\right\}$ is balanced because it is a positive signed graph, a contradiction. As $h=\sum_{i=1}^{s} t_{i}$, the inclusion $V_{\mathbb{X}}(h) \subseteq\left\{\mathbf{v}_{i}\right\}_{i=r+1}^{m}$ follows because char $(K) \neq 2$.

Case (II): $V_{1} \neq \emptyset$ and $V_{2} \neq \emptyset$. If $1 \leq i \leq r$ and $e_{i}$ joins $V_{1}$ and $V_{2}$, then $\sigma\left(e_{i}\right)=+$ and $h\left(\mathbf{v}_{i}\right) \neq 0$ because $\operatorname{char}(K) \neq 2$. Indeed, if $\sigma\left(e_{i}\right)=-$, then $G_{\sigma} \backslash\left\{e_{1}, \ldots, e_{i-1}, e_{i+1}, \ldots, e_{r}\right\}$ is balanced by Theorem [5.1, a contradiction. If $1 \leq i \leq r$ and the two endpoints of $e_{i}$ are both in $V_{1}$ or $V_{2}$, then $\sigma\left(e_{i}\right)=-$ and $h\left(\mathbf{v}_{i}\right) \neq 0$ because $\operatorname{char}(K) \neq 2$. Indeed, if $\sigma\left(e_{i}\right)=+$, then $G_{\sigma} \backslash\left\{e_{1}, \ldots, e_{i-1}, e_{i+1}, \ldots, e_{r}\right\}$ is balanced by Theorem 5.1, a contradiction. Thus, one has the inclusion $V_{\mathbb{X}}(h) \subseteq\left\{\mathbf{v}_{i}\right\}_{i=r+1}^{m}$. If $i>r$, then $h\left(\mathbf{v}_{i}\right)=0$, that is, $\left\{\mathbf{v}_{i}\right\}_{i=r+1}^{m} \subseteq V_{\mathbb{X}}(h)$. This follows noticing that, for $i>r$, one has $\sigma\left(e_{i}\right)=+$ if the endpoints of $e_{i}$ are in $V_{1}$ or $V_{2}$, and $\sigma\left(e_{i}\right)=-$ if $e_{i}$ joins $V_{1}$ and $V_{2}$. Therefore the equality $V_{\mathbb{X}}(h)=\left\{\mathbf{v}_{i}\right\}_{i=r+1}^{m}$ holds.

Now, we show the inequality $r \leq r_{0}$. Pick $h=a_{1} t_{1}+\cdots+a_{s} t_{s}, a_{i}= \pm 1$ for $i=1, \ldots, s$, such that $r_{0}=\left|\mathbb{X} \backslash V_{\mathbb{X}}(h)\right|$. We may assume that the set $\mathbb{X} \backslash V_{\mathbb{X}}(h)$ is equal to $\left\{\mathbf{v}_{1}, \ldots, \mathbf{v}_{r_{0}}\right\}$, and we may also assume that $\left\{e_{1}, \ldots, e_{r_{0}}, \ldots, e_{m}\right\}$ is the set of edges of $G_{\sigma}$ and that $e_{i}$ corresponds to $\mathbf{v}_{i}$ for $i=1, \ldots, m$. It suffices to show that the signed subgraph $H_{\sigma}=G_{\sigma} \backslash\left\{e_{1}, \ldots, e_{r_{0}}\right\}$ is balanced because this implies that $r=\varphi\left(G_{\sigma}\right) \leq r_{0}$. There are disjoint sets $V_{1}$ and $V_{2}$ (possibly empty) such that $V\left(G_{\sigma}\right)=\left\{t_{1}, \ldots, t_{s}\right\}=V_{1} \cup V_{2}$ and

$$
h=\sum_{t_{i} \in V_{1}} t_{i}-\sum_{t_{i} \in V_{2}} t_{i}
$$

Note that $h\left(\mathbf{v}_{i}\right)=0$ if and only if $i>r_{0}$ and $E\left(H_{\sigma}\right)=\left\{e_{i}\right\}_{i=r_{0}+1}^{m}$. If $\sigma\left(e_{i}\right)=-$ for some $i>r_{0}$, then $h\left(\mathbf{v}_{i}\right)=0$, and consequently $e_{i}$ joins $V_{1}$ and $V_{2}$ because $\operatorname{char}(K) \neq 2$. If $\sigma\left(e_{i}\right)=+$ for some $i>r_{0}$, then $h\left(\mathbf{v}_{i}\right)=0$, and consequently the endpoints of $e_{i}$ are in $V_{1}$ or $V_{2}$. Therefore, by Theorem 5.1, $H_{\sigma}=G_{\sigma} \backslash\left\{e_{1}, \ldots, e_{r_{0}}\right\}$ is balanced.

Let $I \neq(0)$ be a graded ideal of $S$ of Krull dimension $k$. The Hilbert function of $S / I$ is:

$$
H_{I}(d):=\operatorname{dim}_{K}\left(S_{d} / I_{d}\right), \quad d=0,1,2, \ldots,
$$


where $I_{d}=I \cap S_{d}$. By a theorem of Hilbert [35, p. 58], there is a unique polynomial $h_{I}(x) \in \mathbb{Q}[x]$ of degree $k-1$ such that $H_{I}(d)=h_{I}(d)$ for $d \gg 0$. The degree of the zero polynomial is -1 .

The degree or multiplicity of $S / I$, denoted $\operatorname{deg}(S / I)$, is the positive integer given by

$$
\operatorname{deg}(S / I):=(k-1) ! \lim _{d \rightarrow \infty} H_{I}(d) / d^{k-1} \text { if } k \geq 1,
$$

and $\operatorname{deg}(S / I)=\operatorname{dim}_{K}(S / I)$ if $k=0$. If $f \in S$, the ideal $(I: f)=\{g \in S \mid g f \in I\}$ is referred to as a colon ideal. Note that $f$ is a zero-divisor of $S / I$ if and only if $(I: f) \neq I$.

Lemma 5.3. [24, Lemma 3.2] Let $\mathbb{X}$ be a finite subset of $\mathbb{P}^{s-1}$ over a field $K$ and let $I(\mathbb{X}) \subset S$ be its vanishing ideal. If $0 \neq f \in S$ is homogeneous and $(I(\mathbb{X}): f) \neq I(\mathbb{X})$, then

$$
\left|V_{\mathbb{X}}(f)\right|=\operatorname{deg}(S /(I(\mathbb{X}), f)) .
$$

The following algebraic formula for the frustration index can be used to compute or estimate this number using Macaulay2 [14] (Example 6.6).

Theorem 5.4. Let $G_{\sigma}$ be a connected unbalanced signed simple graph with frustration index $\varphi\left(G_{\sigma}\right)$ over a field $K$ of $\operatorname{char}(K) \neq 2$, and let $\mathcal{F}$ be the set of linear forms $h=\sum_{i=1}^{s} a_{i} t_{i}$ such that $a_{i}= \pm 1$ for all $i$ and $(I(\mathbb{X}): h) \neq I(\mathbb{X})$. Then

$$
\varphi\left(G_{\sigma}\right)=|\mathbb{X}|-\max \{\operatorname{deg}(S /(I(\mathbb{X}), h)): h \in \mathcal{F}\} .
$$

Proof. The vanishing ideal $I(\mathbb{X})$ does not contains linear forms. This follows by noticing that the incidence matrix of $G_{\sigma}$ has rank equal to $s$, the number of vertices of $G_{\sigma}$, because $G_{\sigma}$ is unbalanced and connected (see Proposition 3.8). Thus $\mathbb{X} \backslash V_{\mathbb{X}}(h) \neq \emptyset$ for any $0 \neq h \in S_{1}$. If $h$ is a linear form, by [12, Lemma 3.1], $V_{\mathbb{X}}(h) \neq \emptyset$ if and only if $(I(\mathbb{X}): h) \neq I(\mathbb{X})$. Therefore, using Lemmas 5.2 and 5.3, we obtain

$$
\begin{aligned}
\varphi\left(G_{\sigma}\right) & =\min \left\{\left|\mathbb{X} \backslash V_{\mathbb{X}}(h)\right|: h=\sum_{i=1}^{s} a_{i} t_{i}, a_{i}= \pm 1 \text { for all } i\right\} \\
& =\min \left\{\left|\mathbb{X} \backslash V_{\mathbb{X}}(h)\right|: h=\sum_{i=1}^{s} a_{i} t_{i}, a_{i}= \pm 1 \text { for all } i \text { and } V_{\mathbb{X}}(h) \neq \emptyset\right\} \\
& =\min \left\{\left|\mathbb{X} \backslash V_{\mathbb{X}}(h)\right|: h \in \mathcal{F}\right\}=|\mathbb{X}|-\max \left\{\left|V_{\mathbb{X}}(h)\right|: h \in \mathcal{F}\right\} \\
& =|\mathbb{X}|-\max \{\operatorname{deg}(S /(I(\mathbb{X}), h)): h \in \mathcal{F}\} .
\end{aligned}
$$

The second equality follows by discarding all $h$ with $V_{\mathbb{X}}(h)=\emptyset$.

Remark 5.5. If we allow the coefficients $a_{1}, \ldots, a_{s}$ to be in $\{0, \pm 1\}$ such that not all of them are zero, we obtain the minimum distance of the incidence matrix code $C$ of $G_{\sigma}$ over any finite field of characteristic $p \neq 2$. This follows from the results of Section 3 and Proposition 5.6 below.

The following algebraic formula for the minimum distance of an incidence matrix code can be used to compute or estimate this number using Macaulay2 [14] and the algorithms of [12, 24].

Proposition 5.6. Let $G_{\sigma}$ be a connected signed simple graph and let $C$ be its incidence matrix code over a finite field $K$. Then the minimum distance of $C$ is given by

$$
\delta(C)=|\mathbb{X}|-\max \left\{\operatorname{deg}(S /(I(\mathbb{X}), h)): h \in S_{1} \backslash I(\mathbb{X}) \text { and }(I(\mathbb{X}): h) \neq I(\mathbb{X})\right\} .
$$

Proof. Let $\mathbf{v}_{1}, \ldots, \mathbf{v}_{m}$ be the column vectors of the incidence matrix of $G_{\sigma}$ and let $P_{i}$ be the point $\left[\mathbf{v}_{i}\right]$ in $\mathbb{P}^{s-1}$ for $i=1, \ldots, m$. Thus, $\mathbb{X}$ is the set of points $\left\{P_{1}, \ldots, P_{m}\right\}$. Note that $C$ is the image of $S_{1}$ - the vector space of linear forms of $S$ - under the evaluation map

$$
\mathrm{ev}_{1}: S_{1} \rightarrow K^{m}, \quad h \mapsto\left(h\left(P_{1}\right), \ldots, h\left(P_{m}\right)\right) .
$$

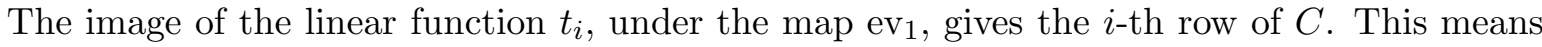
that $C$ is the Reed-Muller-type code $C_{\mathbb{X}}(1)$ in the sense of [13]. The result now follows readily by applying [24, Theorem 4.7]. 


\section{EXAMPLES OF SIGNED GRAPHS}

In this section we illustrate how to use our results in practice with some examples.

Example 6.1. Let $G_{\sigma}$ be a signed simple graph whose underlying graph $G$ is given in Figure 1, let $C$ be the incidence matrix code of $G_{\sigma}$, let $A$ be the incidence matrix of $G_{\sigma}$, and let $M=M[A]$ be the matroid of $C$. Assume that $K$ is either a field of characteristic 2 or that $K$ is any field and $G_{\sigma}=G_{+}$. In either case, by Theorem 3.7(b) and Corollary 3.13(d), $M$ is the cycle matroid of $G$ and, by Proposition 3.8, the rank of $M$ is 10 .

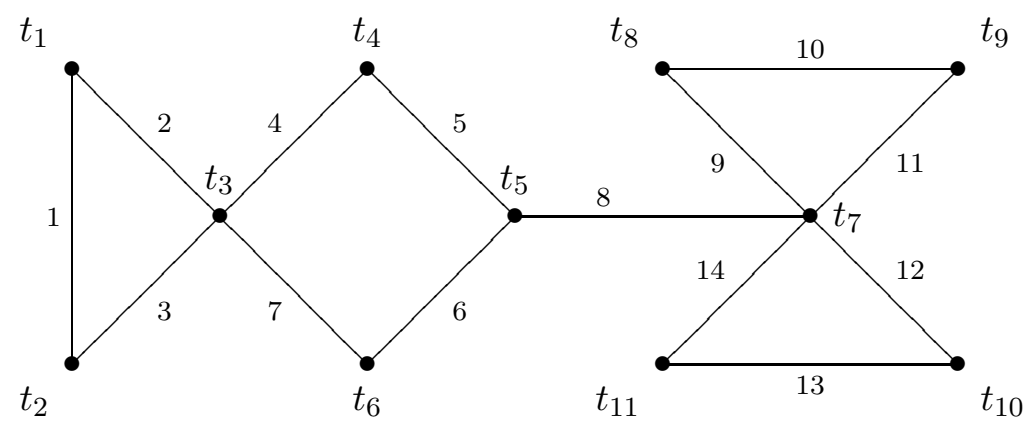

Figure 1. Simple graph $G$ with 11 vertices and 14 edges

Therefore, the circuits of $M$ are the cycles of $G$ and they are given by

$$
c_{1}=\{1,2,3\}, \quad c_{2}=\{9,10,11\}, \quad c_{3}=\{12,13,14\}, \quad c_{4}=\{4,5,6,7\} .
$$

Hence, by applying Theorem 3.19 (a), we get the generalized Hamming weights of $C^{\perp}$ :

\begin{tabular}{|c|c|c|c|c|}
\hline$r$ & 1 & 2 & 3 & 4 \\
\hline$\delta_{r}\left(C^{\perp}\right)$ & 3 & 6 & 9 & 13 \\
\hline
\end{tabular}

Concretely, one has $\delta_{r}\left(C^{\perp}\right)=\left|c_{1} \cup \cdots \cup c_{r}\right|$ for $1 \leq r \leq 4$. Let $R=K\left[x_{1}, \ldots, x_{14}\right]$ be a polynomial ring over the field $K$. The ideal of circuits of $M$ is the squarefree monomial ideal $I=I\left(\mathcal{C}_{M}\right)$ of $R$ generated by all monomials $\prod_{j \in c_{i}} x_{j}$ with $i=1, \ldots, 4$. Using Macaulay2 [14], we obtain that the minimal free resolution of $R / I$ is:

$$
0 \rightarrow R(-13) \rightarrow R(-9) \oplus R^{3}(-10) \rightarrow R^{3}(-6) \oplus R^{3}(-7) \rightarrow R^{3}(-3) \oplus R(-4) \rightarrow R \rightarrow R / I \rightarrow 0
$$

One can verify the values of the $\delta_{r}\left(C^{\perp}\right)$ 's by applying Corollary 4.3 to this resolution. By Wei's duality (Theorem 2.7), one has

\begin{tabular}{|c|c|c|c|c|c|c|c|c|c|c|}
\hline$r$ & 1 & 2 & 3 & 4 & 5 & 6 & 7 & 8 & 9 & 10 \\
\hline$\delta_{r}(C)$ & 1 & 3 & 4 & 5 & 7 & 8 & 10 & 11 & 13 & 14 \\
\hline
\end{tabular}

According to Theorem 3.16, $\delta_{r}(C)=\lambda_{r}(C)$ for $r=1, \ldots, 10$. Removing edge 8 from $G$, we get two connected components. Thus $\delta_{1}(C)=1$. To illustrate the equality $\delta_{7}(C)=10$, note that removing the ten edges that are not in the square of the graph $G$ results in a subgraph with eight connected components, and $\lambda_{7}(C)=10$. The edge biparticity of $G$ is $\varphi\left(G_{-}\right)=3$.

Example 6.2. Let $G$ be the graph of Figure 1, let $K$ be a field of $\operatorname{char}(K) \neq 2$, and let $C$ be the incidence matrix code of $G_{-}$. By Corollary 3.13(c), the circuits of the negative signed graph $G_{-}$, that is, the circuits of the signed-graphic matroid $M\left(G_{-}\right)$, are the even cycles and the bowties of $G$ : 


$$
\begin{aligned}
& c_{1}=\{4,5,6,7\}, c_{2}=\{9,10,11,12,13,14\}, \\
& c_{3}=\{1,2,3,4,5,8,9,10,11\}, c_{4}=\{1,2,3,4,5,8,12,13,14\}, \\
& c_{5}=\{1,2,3,6,7,8,9,10,11\}, c_{6}=\{1,2,3,6,7,8,12,13,14\} .
\end{aligned}
$$

Hence, by Theorem [3.19(b), it follows that $\delta_{r}\left(C^{\perp}\right)=\left|c_{1} \cup \cdots \cup c_{r}\right|$ for $1 \leq r \leq 3$, and we obtain the generalized Hamming weights of $C^{\perp}$ :

\begin{tabular}{|c|c|c|c|}
\hline$r$ & 1 & 2 & 3 \\
\hline$\delta_{r}\left(C^{\perp}\right)$ & 4 & 10 & 14 \\
\hline
\end{tabular}

Let $R=K\left[x_{1}, \ldots, x_{14}\right]$ be a polynomial ring over the field $K$ and let $I=I\left(\mathcal{C}_{M}\right) \subset R$ be the ideal of circuits of the signed-graphic matroid $M\left(G_{-}\right)$. Using Macaulay2 [14], we obtain that the minimal free resolution of $R / I$ is:

$0 \rightarrow R^{4}(-14) \rightarrow R(-10) \oplus R^{4}(-11) \oplus R^{4}(-12) \rightarrow R(-4) \oplus R(-6) \oplus R^{4}(-9) \rightarrow R \rightarrow R / I \rightarrow 0$.

One can verify the values of the $\delta_{r}\left(C^{\perp}\right)$ 's by applying Corollary 4.3 to this resolution. By Wei's duality (Theorem 2.7), we obtain the generalized Hamming weights of $C$ :

\begin{tabular}{|c|c|c|c|c|c|c|c|c|c|c|c|}
\hline$r$ & 1 & 2 & 3 & 4 & 5 & 6 & 7 & 8 & 9 & 10 & 11 \\
\hline$\delta_{r}(C)$ & 2 & 3 & 4 & 6 & 7 & 8 & 9 & 10 & 12 & 13 & 14 \\
\hline
\end{tabular}

According to Theorem 3.16, $\delta_{r}(C)=v_{r}\left(G_{-}\right)$for $r=1, \ldots, 11$. Next we verify these values. Removing edges 2 and 3 from $G$, we get a graph with a bipartite component. Therefore, by Theorem 3.16, $\delta_{1}(C)=2$. To check the other values of $\delta_{r}(C)$ using by Theorem 3.16, note that successively removing from the graph $G$ the edges

$$
\{1,2\}, 3,8,\{10,13\}, 9,11,12,14,\{4,5\}, 6,7 \text {, }
$$

we obtain a subgraph with $r$ bipartite connected components at the $r$-th step. By Theorem 4.7, the regularity of $R / I$ is 11 . The frustration index of $G_{-}$is 3 which is the edge biparticity of $G$.

Example 6.3. Let $G_{\sigma}$ be the signed graph of Figure 2, let $C$ be the incidence matrix code of $G_{\sigma}$ over a finite field of $\operatorname{char}(K)=p \neq 2$, and let $M=M[A]$ be the vector matroid of $C$, where $A$ is the incidence matrix of $G_{\sigma}$.

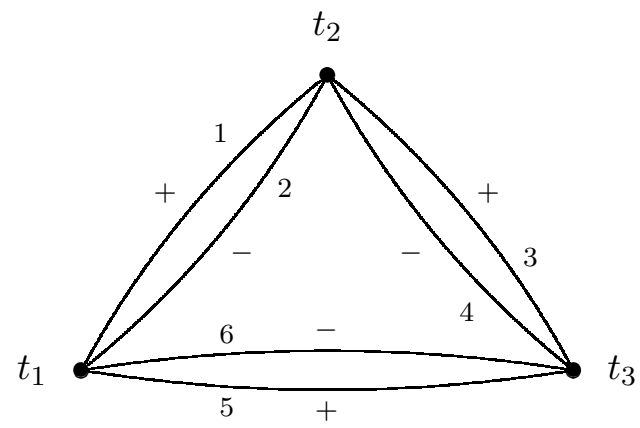

FiguRE 2. Signed graph with 3 vertices and 6 edges

The incidence matrix of the signed graph $G_{\sigma}$ is

$$
A=\left[\begin{array}{cccccc}
1 & 1 & 0 & 0 & 1 & 1 \\
-1 & 1 & 1 & 1 & 0 & 0 \\
0 & 0 & -1 & 1 & -1 & 1
\end{array}\right]
$$


Using Procedure A.1, we obtain the following information. The ideals of circuits and cocircuits of $M$ are given by

$$
\begin{aligned}
I & =\left(x_{1} x_{2} x_{3} x_{4}, x_{1} x_{3} x_{5}, x_{2} x_{4} x_{5}, x_{2} x_{3} x_{6}, x_{1} x_{4} x_{6}, x_{1} x_{2} x_{5} x_{6}, x_{3} x_{4} x_{5} x_{6}\right), \\
I^{*} & =\left(x_{2} x_{4} x_{6}, x_{1} x_{3} x_{6}, x_{1} x_{4} x_{5}, x_{2} x_{3} x_{5}, x_{3} x_{4} x_{5} x_{6}, x_{1} x_{2} x_{5} x_{6}, x_{1} x_{2} x_{3} x_{4}\right),
\end{aligned}
$$

and $\operatorname{reg}(R / I)=\operatorname{reg}\left(R / I^{*}\right)=3$. The generalized Hamming weights of $C^{\perp}$ and $C$ are

\begin{tabular}{|c|c|c|c|}
\hline$r$ & 1 & 2 & 3 \\
\hline$\delta_{r}\left(C^{\perp}\right)$ & 3 & 5 & 6 \\
\hline
\end{tabular}

\begin{tabular}{|c|c|c|c|}
\hline$r$ & 1 & 2 & 3 \\
\hline$\delta_{r}(C)$ & 3 & 5 & 6 \\
\hline
\end{tabular}

Thus, by Theorem 3.16, the cogirth of the signed graph $G_{\sigma}$ is $v_{1}\left(G_{\sigma}\right)=3$, and one has $v_{2}\left(G_{\sigma}\right)=5, v_{3}\left(G_{\sigma}\right)=6$. The frustration index of $G_{\sigma}$ is 3 .

Example 6.4. Let $G_{+}$be the positive signed graph of Figure 3 , let $C$ be the incidence matrix code of $G_{+}$over a finite field $K$, and let $M=M[A]$ be the vector matroid of $C$, where $A$ is the incidence matrix of $G_{+}$. By Corollary 3.13(b), $M$ is the graphic matroid of the underlying graph $G$, that is, the circuits and cocircuits of $M$ are the cycles and cocycles of $G$.

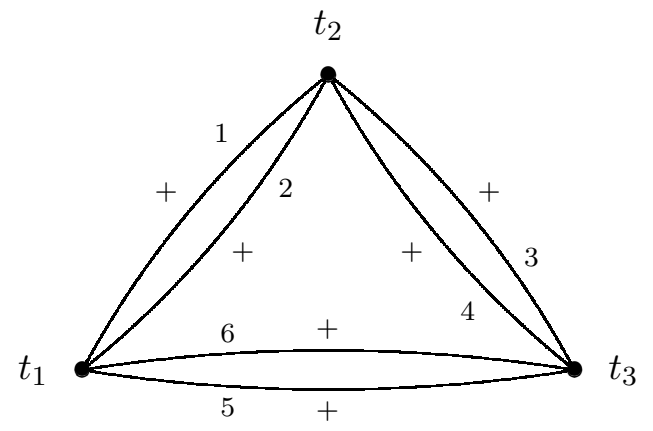

Figure 3. Positive signed graph with 3 vertices and 6 edges

The incidence matrix of the positive signed graph $G_{+}$is

$$
A=\left[\begin{array}{rrrrrr}
1 & 1 & 0 & 0 & 1 & 1 \\
-1 & -1 & 1 & 1 & 0 & 0 \\
0 & 0 & -1 & -1 & -1 & -1
\end{array}\right]
$$

Using Procedure A.2, we obtain the following information. The ideals of circuits and cocircuits of $M$ are given by

$$
\begin{aligned}
I & =\left(x_{5} x_{6}, x_{3} x_{4}, x_{1} x_{2}, x_{2} x_{4} x_{6}, x_{1} x_{4} x_{6}, x_{2} x_{3} x_{6}, x_{1} x_{3} x_{6}, x_{2} x_{4} x_{5}, x_{1} x_{4} x_{5}, x_{2} x_{3} x_{5}, x_{1} x_{3} x_{5}\right), \\
I^{*} & =\left(x_{3} x_{4} x_{5} x_{6}, x_{1} x_{2} x_{5} x_{6}, x_{1} x_{2} x_{3} x_{4}\right),
\end{aligned}
$$$$
\operatorname{reg}(R / I)=2 \text {, and } \operatorname{reg}\left(R / I^{*}\right)=4 \text {. The generalized Hamming weights of } C^{\perp} \text { and } C \text { are }
$$

\begin{tabular}{|c|c|c|c|c|}
\hline$r$ & 1 & 2 & 3 & 4 \\
\hline$\delta_{r}\left(C^{\perp}\right)$ & 2 & 4 & 5 & 6 \\
\hline
\end{tabular}$\quad$\begin{tabular}{|c|c|c|}
\hline$r$ & 1 & 2 \\
\hline$\delta_{r}(C)$ & 4 & 6 \\
\hline
\end{tabular}

Thus, by Theorem 3.16, the edge connectivity of $G$ is $\lambda_{1}(G)=4$, and $\lambda_{2}(G)=6$.

Example 6.5. Let $G_{-}$be the negative signed graph of Figure 4 , let $C$ be the incidence matrix code of $G_{-}$over a field $K$ of characteristic $p \neq 2$, and let $M=M[A]$ be the vector matroid of $C$, where $A$ is the incidence matrix of $G_{-}$. By Corollary $3.15, M$ is the even cycle matroid of the underlying graph $G$, that is, the circuits of $M$ are the even cycles and bowties of $G$. 


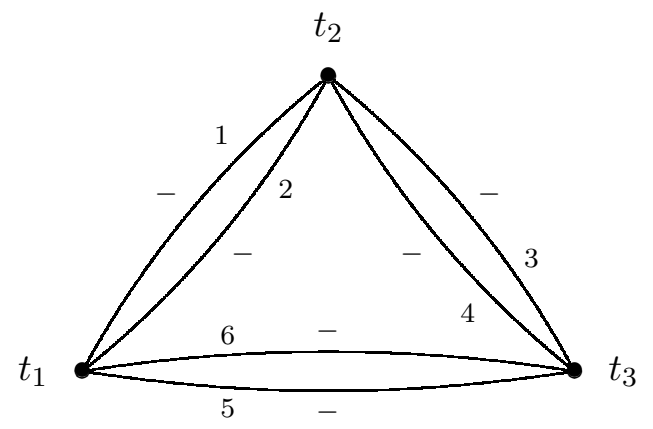

Figure 4. Negative signed graph with 3 vertices and 6 edges

The incidence matrix of the negative signed graph $G_{-}$is the incidence matrix of $G$ :

$$
A=\left[\begin{array}{llllll}
1 & 1 & 0 & 0 & 1 & 1 \\
1 & 1 & 1 & 1 & 0 & 0 \\
0 & 0 & 1 & 1 & 1 & 1
\end{array}\right]
$$

Using Procedure A.3, we obtain the following information. The ideals of circuits and cocircuits of $M$ are given by

$$
I=\left(x_{1} x_{2}, x_{3} x_{4}, x_{5} x_{6}\right), \quad I^{*}=\left(x_{1} x_{2}, x_{3} x_{4}, x_{5} x_{6}\right),
$$

$\operatorname{reg}(R / I)=\operatorname{reg}\left(R / I^{*}\right)=3$. The generalized Hamming weights of $C^{\perp}$ and $C$ are

\begin{tabular}{|c|c|c|c|}
\hline$r$ & 1 & 2 & 3 \\
\hline$\delta_{r}\left(C^{\perp}\right)$ & 2 & 4 & 6 \\
\hline
\end{tabular}$\quad$\begin{tabular}{|c|c|c|c|}
\hline$r$ & 1 & 2 & 3 \\
\hline$\delta_{r}(C)$ & 2 & 4 & 6 \\
\hline
\end{tabular}

Thus, by Theorem 3.16, the cogirth of $G_{-}$is $v_{1}\left(G_{-}\right)=2$, and $v_{2}\left(G_{-}\right)=4, v_{3}\left(G_{-}\right)=6$.

Example 6.6. Let $G_{\sigma}$ be the signed graph of Figure 5 and let $G$ be its underlying graph. The incidence matrix of $G_{\sigma}$ is given in Procedure A.4. Using this procedure we obtain that the frustration index $\varphi\left(G_{\sigma}\right)$ of $G_{\sigma}$ is 7 and the frustration index $\varphi\left(G_{-}\right)$of the negative signed graph $G_{-}$is 6 . The minimum distance $\delta(C)$ of the incidence matrix code $C$ of $G_{\sigma}$ is 4 if char $(K) \neq 2$ and $\delta(C)$ is 3 if $\operatorname{char}(K)=2$. In this case $\delta(C)=\delta\left(C^{\perp}\right)$ in any characteristic.

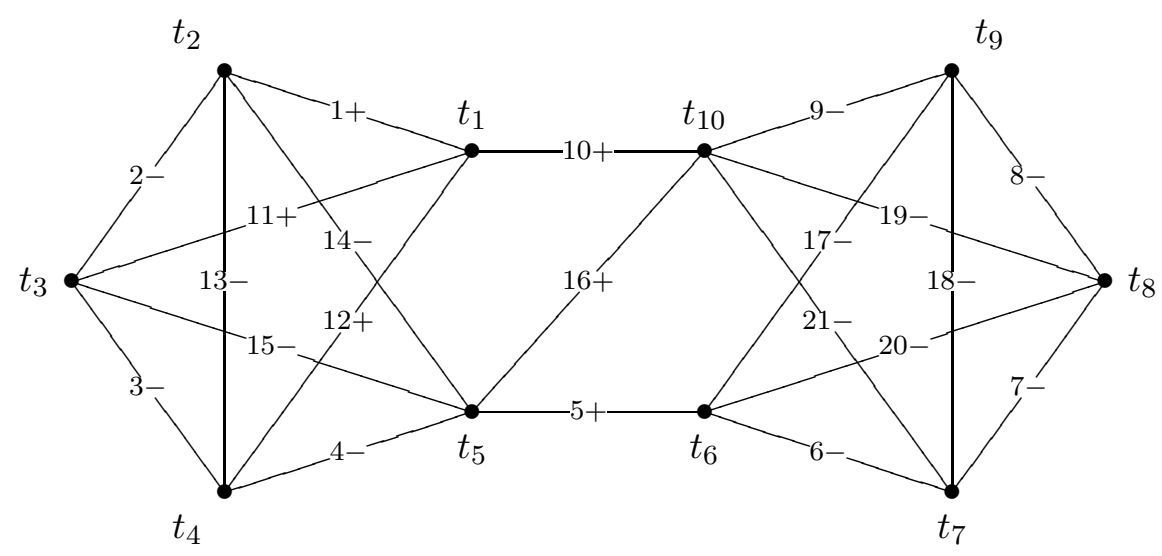

FiguRE 5. Unbalanced signed graph with frustration index 7 


\section{ACKNOWLEDGMENTS}

We thank Thomas Zaslavsky for suggesting to generalize our work on incidence matrix codes of graphs to signed graphs, and for pointing out that the edge biparticity of a graph is a special case of the frustration index of a signed graph. Computations with Macaulay2 [14, Matroids [8], and SageMath [30] were important to verifying and computing examples given in this paper.

\section{Appendix A. Procedures for Macaulay2 and Matroids}

In this section we give procedures for Macaulay2 [14], using the field of rational numbers as the ground field, to compute the generalized Hamming weights of the incidence matrix code of a signed graph and the corresponding graph theoretical invariants ( $r$-th cogirth, $r$-th edge connectivity), as well as the ideals of circuits, cocircuits, cycles and cocycles of a signed graph, and their algebraic invariants (Betti numbers, shifts, regularity). We also give a procedure to compute the frustration index of a connected signed simple graph. In all procedures the input is a rational matrix. The package Matroids [8] plays an important role here because it computes the circuits and cocircuits of a vector matroid over the field $\mathbb{Q}$ of rational numbers.

Procedure A.1. Given the incidence matrix $A$ of a signed graph $G_{\sigma}$ over a field of $\operatorname{char}(K) \neq 2$, the procedure below computes the following:

- The ideal of circuits and the ideal of cocircuits of $G_{\sigma}$, and its regularity.

- The graded Betti numbers of the ideal of circuits and the ideal of cocircuits of $G_{\sigma}$.

- The weight hierarchies of the incidence matrix code $C$ of $G_{\sigma}$ and of its dual code $C^{\perp}$.

- The $r$-th cogirth of $G_{\sigma}$ (Theorem 3.16).

The next procedure corresponds to Example 6.3. To compute other examples just change the incidence matrix $A$.

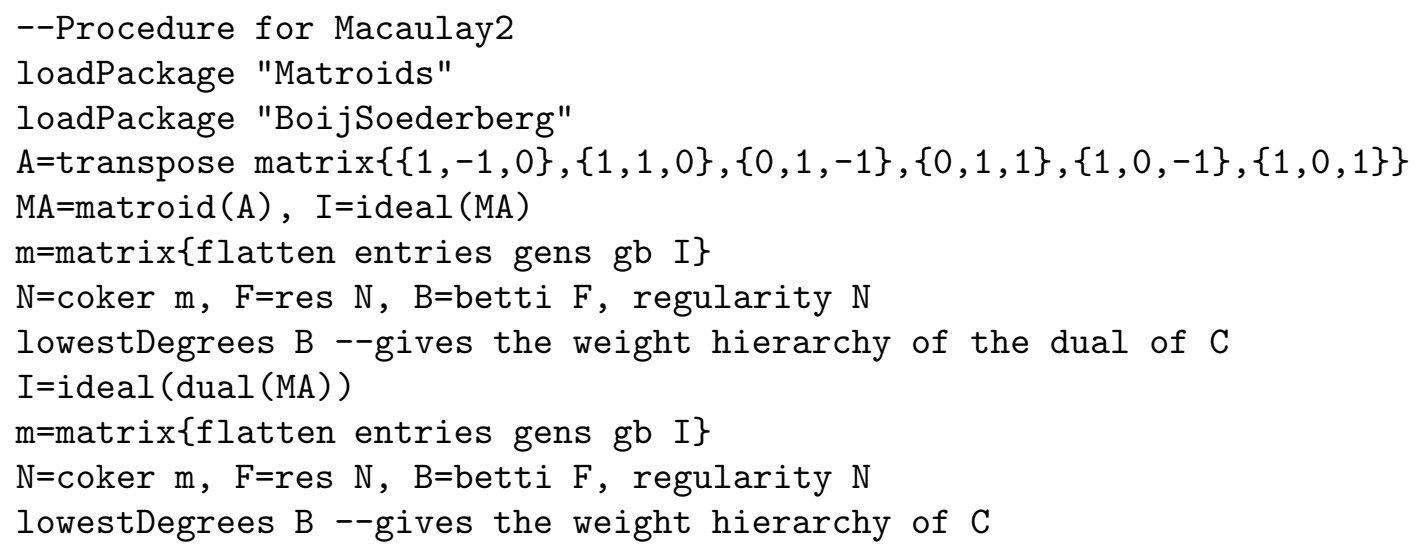

Procedure A.2. Using the incidence matrix $A$ of a positive signed graph $G_{+}$over a field $K$ and the Procedure A.1, we can compute the following:

- The ideal of cycles and the ideal of cocycles of $G$ and its regularity.

- The graded Betti numbers of the ideals of cycles and cocycles.

- The weight hierarchies of the incidence matrix code of $G_{+}$and of its dual code, and the generalized Hamming weights of the incidence matrix code of a digraph $\mathcal{D}$.

- The $r$-th edge connectivity of $G$.

The next incidence matrix corresponds to Example 6.4. 
--Incidence matrix for Macaulay2

$A=\operatorname{transpose} \operatorname{matrix}\{\{1,-1,0\},\{1,-1,0\},\{0,1,-1\},\{0,1,-1\},\{1,0,-1\},\{1,0,-1\}\}$

Procedure A.3. Using the incidence matrix $A$ of a negative signed graph $G_{-}$over a field $K$ of characteristic $p \neq 2$ and the Procedure A.1, we can compute the following:

- The ideal $I$ of the even cycles and bowties of $G$ and the ideal $I^{*}$ of cocircuits of $G_{-}$.

- The graded Betti numbers of $I$ and $I^{*}$, and its regularity.

- The weight hierarchies of the incidence matrix code of $G_{-}$and of its dual code.

- The $r$-th cogirth of $G_{-}$.

The next incidence matrix corresponds to Example 6.5.

--Incidence matrix for Macaulay2

$A=\operatorname{transpose} \operatorname{matrix}\{\{1,1,0\},\{1,1,0\},\{0,1,1\},\{0,1,1\},\{1,0,1\},\{1,0,1\}\}$

Procedure A.4. One can use Theorem 5.4 and Macaulay2 [14] to compute the frustration index of a connected unbalanced signed simple graph $G_{\sigma}$. The incidence matrix of the following procedure corresponds to the graph of Figure 5 given in Example 6.6.

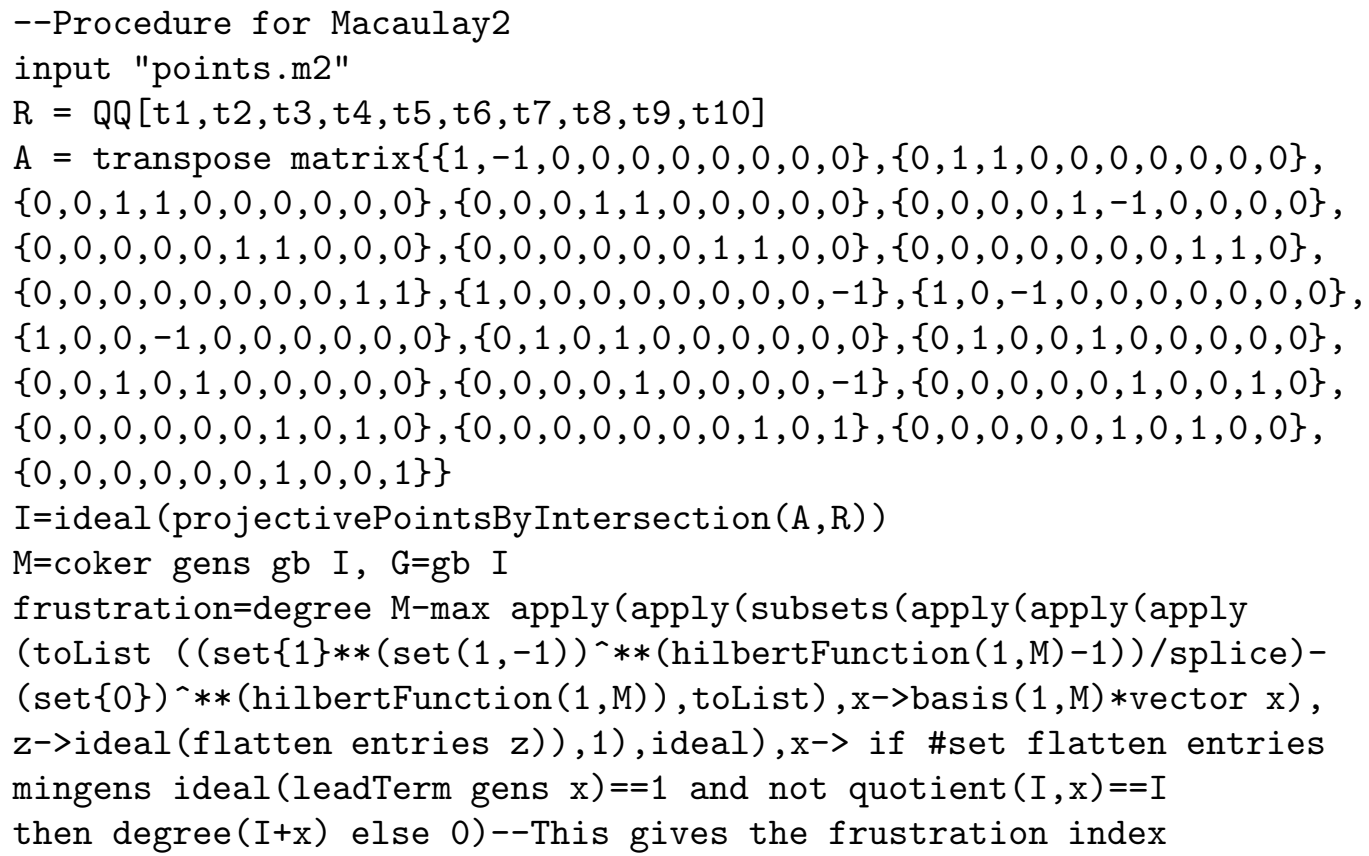

\section{REFERENCES}

[1] B. Anzis, M. Garrousian and S. Tohăneanu, Generalized star configurations and the Tutte polynomial, J. Algebraic Combin. 46 (2017), no. 1, 165-187.

[2] I. Bermejo, P. Gimenez and A. Simis, Polar syzygies in characteristic zero: the monomial case, J. Pure Appl. Algebra 213 (2009), 1-21.

[3] T. Britz, MacWilliams identities and matroid polynomials, Electron. J. Combin. 9 (2002), no. 1, Paper 19, $16 \mathrm{pp}$.

[4] T. Britz, Higher support matroids, Discrete Math. 307 (2007), no. 17-18, 2300-2308.

[5] D. Britz, T. Britz, K. Shiromoto and H. K. Sørensen, The higher weight enumerators of the doubly-even, self-dual [48,24,12] code, IEEE Trans. Inform. Theory 53 (2007), no. 7, 2567-2571.

[6] T. Britz, T. Johnsen, D. Mayhew and K. Shiromoto, Wei-type duality theorems for matroids, Des. Codes Cryptogr. 62 (2012), no. 3, 331-341. 
[7] T. Brylawski, Appendix of matroid cryptomorphisms, Theory of matroids, pp. 298-316, Encyclopedia Math. Appl., 26, Cambridge Univ. Press, Cambridge, 1986.

[8] J. Chen, Matroids, A package for computations with matroids, version 0.9.6, 2018.

[9] P. Dankelmann, J. D. Key and B. G. Rodrigues, Codes from incidence matrices of graphs, Des. Codes Cryptogr. 68 (2013), no. 1-3, 373-393.

[10] D. Eisenbud, The geometry of syzygies: A second course in commutative algebra and algebraic geometry, Graduate Texts in Mathematics 229, Springer-Verlag, New York, 2005.

[11] I. Gitler and R. H. Villarreal, Graphs, Rings and Polyhedra, Aportaciones Mat. Textos, 35, Soc. Mat. Mexicana, México, 2011.

[12] M. González-Sarabia, J. Martínez-Bernal, R. H. Villarreal and C. E. Vivares, Generalized minimum distance functions, J. Algebraic Combin., to appear.

[13] M. González-Sarabia, C. Rentería and H. Tapia-Recillas, Reed-Muller-type codes over the Segre variety, Finite Fields Appl. 8 (2002), no. 4, 511-518.

[14] D. Grayson and M. Stillman, Macaulay2, 1996. Available via anonymous ftp from math.uiuc.edu.

[15] F. Harary, On the notion of balance of a signed graph, Michigan Math. J. 2 (1953-1954), $143-146$.

[16] P. Heijnen and R. Pellikaan, Generalized Hamming weights of $q$-ary Reed-Muller codes, IEEE Trans. Inform. Theory 44 (1998), no. 1, 181-196.

[17] T. Helleseth, T. Kløve and J. Mykkeltveit, The weight distribution of irreducible cyclic codes with block length $n_{1}\left(\left(q^{l}-1\right) / N\right)$, Discrete Math. 18 (1977), no. 2, 179-211.

[18] T. Johnsen, J. Roksvold and H. Verdure, A generalization of weight polynomials to matroids, Discrete Math. 339 (2016), no. 2, 632-645.

[19] T. Johnsen and H. Verdure, Hamming weights and Betti numbers of Stanley-Reisner rings associated to matroids, Appl. Algebra Engrg. Comm. Comput. 24 (2013), no. 1, 73-93.

[20] T. Johnsen and H. Verdure, Generalized Hamming weights for almost affine codes, IEEE Trans. Inform. Theory 63 (2017), no. 4, 1941-1953.

[21] L. Khachiyan, E. Boros, K. Elbassioni, V. Gurvich and K. Makino, On the complexity of some enumeration problems for matroids, SIAM J. Discrete Math. 19 (2005), no. 4, 966-984.

[22] T. Kløve, Support weight distribution of linear codes, Discrete Math. 106/107 (1992), 311-316.

[23] F. J. MacWilliams and N. J. A. Sloane, The Theory of Error-correcting Codes, North-Holland, 1977.

[24] J. Martínez-Bernal, Y. Pitones and R. H. Villarreal, Minimum distance functions of graded ideals and ReedMuller-type codes, J. Pure Appl. Algebra 221 (2017), 251-275.

[25] J. Martínez-Bernal and R. H. Villarreal, Toric ideals generated by circuits, Algebra Colloq. 19 (2012), no. 4, 665-672.

[26] J. Martínez-Bernal, M. A. Valencia-Bucio and R. H. Villarreal, Generalized Hamming weights of projective Reed-Muller-type codes over graphs, Discrete Math., to appear.

[27] W. Olaya-León and C. Granados-Pinzón, The second generalized Hamming weight of certain Castle codes, Des. Codes Cryptogr. 76 (2015), no. 1, 81-87.

[28] J. Oxley, Matroid Theory, Oxford University Press, Oxford, 1992.

[29] R. T. Rockafellar, The elementary vectors of a subspace of $R^{N}$, in Combinatorial Mathematics and its Applications, Proc. Chapel Hill Conf., Univ. North Carolina Press, 1969, pp. 104-127.

[30] SageMath, the Sage Mathematics Software System (Version 8.4), The Sage Developers, 2018, available from http://www.sagemath.org.

[31] H. G. Schaathun and W. Willems, A lower bound on the weight hierarchies of product codes, Discrete Appl. Math. 128 (2003), no. 1, 251-261.

[32] A. Simis, W. V. Vasconcelos and R. H. Villarreal, The integral closure of subrings associated to graphs, J. Algebra 199 (1998), 281-289.

[33] J. M. S. Simões-Pereira, On matroids on edge sets of graphs with connected subgraphs as circuits II, Discrete Math. 12 (1975), 55-78.

[34] P. Solé and T. Zaslavsky, A coding approach to signed graphs, SIAM J. Discrete Math. 7 (1994), no. 4, $544-553$.

[35] R. Stanley, Hilbert functions of graded algebras, Adv. Math. 28 (1978), 57-83.

[36] R. Stanley, Combinatorics and Commutative Algebra, Birkhäuser Boston, 2nd ed., 1996.

[37] M. Tsfasman, S. Vladut and D. Nogin, Algebraic geometric codes: basic notions, Mathematical Surveys and Monographs 139, American Mathematical Society, Providence, RI, 2007.

[38] A. Vardy, Algorithmic complexity in coding theory and the minimum distance problem, STOC '97 (El Paso, TX), 92-109, ACM, New York, 1999. 
[39] R. H. Villarreal, Rees algebras of edge ideals, Comm. Algebra 23 (1995), 3513-3524.

[40] R. H. Villarreal, Monomial algebras and polyhedral geometry, in Handbook of Algebra, Vol. 3 (M. Hazewinkel Ed.), Elsevier, Amsterdam, 2003, pp. 257-314.

[41] R. H. Villarreal, Monomial Algebras, Second Edition, Monographs and Research Notes in Mathematics, Chapman and Hall/CRC, 2015.

[42] V. K. Wei, Generalized Hamming weights for linear codes, IEEE Trans. Inform. Theory 37 (1991), no. 5, $1412-1418$.

[43] V. K. Wei and K. Yang, On the generalized Hamming weights of product codes, IEEE Trans. Inform. Theory 39 (1993), no. 5, 1709-1713.

[44] D. J. A. Welsh, Matroid Theory, L. M. S. Monographs, no. 8, Academic Press, London-New York, 1976.

[45] M. Yang, J. Lin, K. Feng and D. Lin, Generalized Hamming weights of irreducible cyclic codes, IEEE Trans. Inform. Theory 61 (2015), no. 9, 4905-4913.

[46] T. Zaslavsky, Signed graphs, Discrete Appl. Math. 4 (1982), no. 1, 47-74. Erratum, Discrete Appl. Math. 5 (1983), no. 2, 248.

[47] T. Zaslavsky, Biased graphs. I. Bias, balance, and gains, J. Combin. Theory Ser. B 47 (1989), no. 1, 32-52.

[48] T. Zaslavsky, Biased graphs. II. The three matroids, J. Combin. Theory Ser. B 51 (1991), no. 1, 46-72.

[49] T. Zaslavsky, Glossary of signed and gain graphs and allied areas, Electron. J. Combin. 5 (1998), Dynamic Surveys 9, $41 \mathrm{pp}$.

Departamento de Matemáticas, Centro de Investigación y de Estudios Avanzados del IPN, Apartado Postal 14-740, 07000 Mexico City, Mexico.

E-mail address: jmb@math.cinvestav.mx

Departamento de Matemáticas, Centro de Investigación y de Estudios Avanzados del IPN, Apartado Postal 14-740, 07000 Mexico City, Mexico

E-mail address: mavalencia@math.cinvestav.mx

Departamento de Matemáticas, Centro de Investigación y de Estudios Avanzados del IPN, Apartado Postal 14-740, 07000 Mexico City, Mexico

E-mail address: vila@math.cinvestav.mx 